\title{
Different pole structures in line shapes of the $X(3872)$
}

\author{
Xian-Wei Kang, J. A. Oller ${ }^{\mathrm{a}}$ \\ Departamento de Física, Universidad de Murcia, 30071 Murcia, Spain
}

Received: 2 January 2017 / Accepted: 31 May 2017 / Published online: 15 June 2017

(C) The Author(s) 2017. This article is an open access publication

\begin{abstract}
We introduce a near-threshold parameterization that is more general than the effective-range expansion up to and including the effective range because it can also handle a near-threshold zero in the $D^{0} \bar{D}^{* 0} S$-wave. In terms of it we analyze the CDF data on inclusive $p \bar{p}$ scattering to $J / \psi \pi^{+} \pi^{-}$, and the Belle and BaBar data on $B$ decays to $K J / \psi \pi^{+} \pi^{-}$and $K D \bar{D}^{* 0}$ around the $D^{0} \bar{D}^{* 0}$ threshold. It is shown that data can be reproduced with similar quality for $X(3872)$ being a bound and/or a virtual state. We also find that $X(3872)$ might be a higher-order virtual-state pole (double or triplet pole), in the limit in which the small $D^{* 0}$ width vanishes. Once the latter is restored the corrections to the pole position are non-analytic and much bigger than the $D^{* 0}$ width itself. The $X(3872)$ compositeness coefficient in $D^{0} \bar{D}^{* 0}$ ranges from nearly 0 up to 1 in the different scenarios.
\end{abstract}

\section{Introduction}

The $X(3872)$ has been analyzed in great phenomenological detail by employing $S$-wave effective-range-expansion (ERE) parameterizations in Refs. [1-3]. References [2,3] includes only the $D \bar{D}^{*}$ scattering length, $a$, while Ref. [1] also includes the effective-range $(r)$ contribution. ${ }^{1}$ A detailed comparison between both approaches is given in Sect. 6 of Ref. [3]. Indeed, the use of the ERE up to and including the effective range is more general than employing a Flatté parameterization (also used in Refs. [4-6]), because only negative effective ranges can be generated within the latter [7]. ${ }^{2}$

\footnotetext{
$\overline{1}$ To shorten the presentation we actually refer by $D \bar{D}^{*}$ to the $C=+$ combination $\left(D \bar{D}^{*}+\bar{D} D^{*}\right) / \sqrt{2}$.

${ }^{2}$ As follows from Ref. [8], the Flatté parameterization and the ERE including the effective-range contribution are equivalent if the former is written in terms of the bare mass and coupling squared that need to be tuned, taking a priori any sign, to reproduce the values of the residue and pole position of the partial wave.

a e-mail: oller@um.es
}

However, the ERE convergence radius might be severely limited due to the presence of near-threshold zeros of the partial wave, in this case the $D^{0} \bar{D}^{* 0} S$-wave. These zeros, also called Castillejo-Dalitz-Dyson (CDD) poles [9], constitute the major criticism to apply Weinberg's compositeness theorem to evaluate the actual compositeness of a near-threshold bound state [10], because it is based on the ERE up to the effective-range contribution. ${ }^{3}$ The same criticism is of course applicable to the papers $[1-3,5,6]$ referred in the previous paragraph.

The issue about the possible presence of near-threshold zero in the $S$-wave partial wave and the spoil of the corresponding ERE was also discussed more recently in Ref. [11]. One of the main conclusions of this reference was that in order to end with a near-threshold zero one needs also three shallow poles. In this way this situation was qualified as highly accidental by the authors of Ref. [11]. However, this conclusion is not necessarily correct, that is, one can have a near-threshold zero with only two nearby poles, without the need of a third one. The reason for this misstep in the study of Ref. [11] was a misuse of the relation between the position of the zero and the location of the poles in the three-momentum complex plane, as we discuss in detail in Sect. 6.3. Twocoupled-channels effects were included in Ref. [12] along the similar lines of mixing the exchange of a resonance with direct interactions between the mesons, in the limit of validity of the scattering length approximation for the latter ones. In turn, the coupled-channel generalization of Ref. [11] was derived in Ref. [13]. In the energy region around the $D^{0} \bar{D}^{* 0}$ threshold where $X(3872)$ sits, the coupled-channel results of Refs. [12,13] reduce to a partial wave whose structure can be deduced from the elastic one-channel $D^{0} \bar{D}^{* 0}$ scatter-

\footnotetext{
3 This serious criticism was originally due to R. Blankenbecler, M. L. Goldberger, K. Johnson and S. B. Treiman, as explicitly stated in the note added in proof in Ref. [10], warning about the possible presence of CDD poles for $E>-B$ in the Low equation used in this reference. The only way to skip this problem is to ascertain the range of convergence of the ERE, typically from data.
} 
ing, because the $D^{+} D^{*-}$ threshold is relatively much further away. We also indicate here that Ref. [11] cannot reproduce positive values for the $D^{0} \bar{D}^{* 0} S$-wave effective range, while our approach is more general in this respect and can also give rise to positive values of this low-energy scattering parameter. These two points are also shown explicitly below.

As in Refs. [1-3] we avoid any explicit dynamical model for the $D \bar{D}^{*}$ dynamics to study the $X(3872)$ line shapes in the BaBar $[14,15]$ and Belle $[16,17]$ data on the $B$ decays to $K^{ \pm} J / \psi \pi^{+} \pi^{-}$and $K J / \psi D^{0} \bar{D}^{* 0}$. In addition, we also consider the higher-statistics data from the inclusive $p \bar{p}$ scattering to $J / \psi \pi^{+} \pi^{-}$measured by the CDF Collaboration [18] and that gives rise to a more precise determination of the mass of $X$ (3872) [19]. However, we employ a more general parameterization than the ERE expansion up to and including the effective-range contribution by explicitly taking into account the possibility of the presence of a CDD pole very close to the $D^{0} \bar{D}^{* 0}$ threshold. Our formalism has as limiting cases those of Refs. [1-3], but it can also consider other cases. In particular, while in Refs. [1-3] X (3872) turns out to be either a bound or a virtual-state pole, we also find other qualitatively different scenarios that can reproduce data with similar quality as well. In two of these new situations the $X(3872)$ is simultaneously a bound and a virtual state and for one of them the $D^{0} \bar{D}^{* 0}$ compositeness coefficient is just of a few per cent. This is also an interesting counterexample for the conclusions of Ref. [11], because it has a CDD pole almost on top of threshold with only two shallow poles. Remarkably, we also find other cases with two/three virtual-states poles, such that in the limit of vanishing width of $D^{* 0}$ these poles become degenerate and result in a second/third-order $S$-matrix pole. Along the lines of the discussions, we also match our resulting partial-wave amplitude from $S$-matrix theory with the one deduced in Ref. [11] in terms of the exchange of a bare state and direct interactions between the $D^{0} \bar{D}^{* 0}$ mesons. Similarly, this is also done with the onechannel reduction of Ref. [12] in the $D^{0} \bar{D}^{* 0}$ near-threshold region.

The paper is organized as follows. After this Introduction we present the formalism for the analysis of the line shapes of the $X(3872)$ in Sects. 2-5. The different scenarios and their characteristics are the main subject of Sect. 6, where we also give the numerical results of the fits in each case, the poles obtained and their properties. After the concluding remarks in Sect. 7, we give some more technical and detailed material in Appendices A, B and C.

\section{$2 J / \psi \pi^{+} \pi^{-}$partial-decay rate and differential cross section}

For the decay $B \rightarrow K F$ through $X(3872)$ we have the decay chain $B \rightarrow K X$ and then $X \rightarrow F$. We can write the decay
Fig. 1 Skeleton Feynman diagram for the $B \rightarrow K F$ decay through the $X(3872)$ resonance

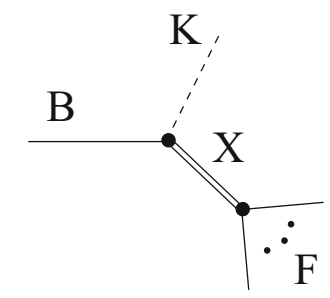

amplitude $T_{F}$, represented schematically by the Feynman diagram in Fig. 1, as

$T_{F}=-\frac{\mathcal{V}_{L} \mathcal{V}_{X}}{Q^{2}-P_{X}^{2}}$,

where $\mathcal{V}_{L}$ and $\mathcal{V}_{X}$ refer to the vertices from left to right in Fig. 1, $Q^{2}$ is the invariant mass squared of the subsystem of final particles $F$ (which also coincides with the invariant mass squared of the $X(3872)$ resonance) and the $X(3872)$ pole position is $P_{X}=M_{X}-i \Gamma_{X} / 2$, with $M_{X}$ and $\Gamma_{X}$ its mass and width, respectively. The partial-decay width for this process is

$$
\begin{aligned}
\Gamma_{B \rightarrow K F}= & \frac{1}{2 M_{B}} \int(2 \pi)^{4} \delta\left(P-Q-p_{k}\right) \frac{\mathrm{d}^{3} p_{K}}{(2 \pi)^{3} 2 E_{K}} \mathrm{~d} \mathcal{F} \\
& \times \frac{\left|\mathcal{V}_{L}\right|^{2}\left|\mathcal{V}_{X}\right|^{2}}{\left|Q^{2}-P_{X}^{2}\right|^{2}}
\end{aligned}
$$

In this equation, $P$ is the total four-momentum of the system (or that of the $B$ meson), $p_{K}$ is the four-momentum of the kaon and $Q$ is the one of $X$ (3872) (or $F$ subsystem). Let us denote by a subscript $i$ (with $i=1, \ldots, N_{F}$ ) the particles in $F$ and denote the four-momentum of every particle as $p_{i}$, so that $Q=\sum_{i=1}^{N_{F}} p_{i}$. Then we define $\mathrm{d} \mathcal{F}$ as the count of states in the subsystem $F$,

$\mathrm{d} \mathcal{F}=\prod_{i=1}^{N_{F}} \frac{\mathrm{d}^{3} p_{i}}{(2 \pi)^{3} 2 E_{i}}$,

being $E_{i}=\sqrt{m_{i}^{2}+\mathbf{p}_{i}^{2}}$ the energy of the $i$ th particle with mass $m_{i}$ and three-momentum $\mathbf{p}_{i}$. The phase space factor for $\mathcal{F}$, which we denote by d $\mathfrak{f}$, can be obtained by extracting from $\mathrm{d} \mathcal{F}$ its total four-momentum contribution, so that

$\mathrm{d} \mathcal{F}=\frac{\mathrm{d}^{4} Q}{(2 \pi)^{4}} \mathrm{~d} \mathfrak{f}$.

We take this into the expression for $\Gamma_{B \rightarrow K F}$, Eq. (2), and multiply and divide the integrand by $Q_{0}=+\sqrt{Q^{2}+\mathbf{Q}^{2}}$, which is the energy corresponding to a particle of mass $\sqrt{Q^{2}}$ and three-momentum squared $\mathbf{Q}^{2}$. Notice that $Q_{0}>0$ and $Q^{2}>0$ because they are the total energy and invariant mass squared, in order, of the asymptotic particles in $F$. We then have 


$$
\begin{aligned}
\Gamma_{B \rightarrow K F}= & \int \frac{\mathrm{d} Q_{0}}{2 \pi} \mathrm{d} \mathfrak{f} 2 \sqrt{Q^{2}+\mathbf{Q}^{2}} \frac{\left|\mathcal{V}_{X}\right|^{2}}{\left|Q^{2}-P_{X}^{2}\right|^{2}} \\
& \times \frac{1}{2 M_{B}} \int(2 \pi)^{4} \delta\left(P-Q-p_{K}\right)\left|\mathcal{V}_{L}\right|^{2} \\
& \times \frac{\mathrm{d}^{3} p_{K}}{(2 \pi)^{3} 2 E_{K}} \frac{\mathrm{d}^{3} Q}{(2 \pi)^{3} 2 \sqrt{Q^{2}+\mathbf{Q}^{2}}} .
\end{aligned}
$$

The decay width of a $B$ meson into a kaon $K$ and a resonance $X$ of mass $\sqrt{Q^{2}}, \Gamma_{B \rightarrow K X}\left(Q^{2}\right)$, is the term on the right-hand side of the second line in the previous equation:

$$
\begin{aligned}
\Gamma_{B \rightarrow K X}\left(Q^{2}\right)= & \frac{1}{2 M_{B}} \int(2 \pi)^{4} \delta\left(P-Q-p_{K}\right)\left|\mathcal{V}_{L}\right|^{2} \\
& \times \frac{\mathrm{d}^{3} p_{K}}{(2 \pi)^{3} 2 E_{K}} \frac{\mathrm{d}^{3} Q}{(2 \pi)^{3} 2 \sqrt{Q^{2}+\mathbf{Q}^{2}}} .
\end{aligned}
$$

Similarly the decay width of $X\left(\sqrt{Q^{2}}\right)$ into $F, \Gamma_{X \rightarrow F}\left(Q^{2}\right)$ is given by

$$
\Gamma_{X \rightarrow F}\left(Q^{2}\right)=\frac{1}{2 \sqrt{Q^{2}}} \int \mathrm{d} \mathfrak{f}\left|\mathcal{V}_{X}\right|^{2} .
$$

We also perform the change of variables from $Q_{0}$ to $Q^{2}$, related by

$$
Q^{2}=Q_{0}^{2}-\mathbf{Q}^{2} \text {. }
$$

Then, in terms of Eqs. (6) and (7), we can rewrite Eq. (5) as

$$
\Gamma_{B \rightarrow K F}=\int \frac{\mathrm{d} Q^{2}}{2 \pi} 2 \sqrt{Q^{2}} \frac{\Gamma_{B \rightarrow K X}\left(Q^{2}\right) \Gamma_{X \rightarrow F}\left(Q^{2}\right)}{\left|Q^{2}-P_{X}^{2}\right|^{2}} .
$$

One can formulate more conveniently the previous expression by noticing that we are interested in event distributions with invariant mass around the nominal mass of $X$ (3872) and $\Gamma_{X} \ll M_{X}\left(\Gamma_{X}<1.2 \mathrm{MeV}[19]\right)$. We then approximate

$Q^{2}-P_{X}^{2} \simeq 2 \sqrt{Q^{2}}\left(\sqrt{Q^{2}}-P_{X}\right)$,

in the propagator of $X$ (3872) in Eq. (9). Measuring the invariant mass of $X(3872)$ with respect to the $D^{0} \bar{D}^{* 0}$ threshold, we define the energy variable $E$ as

$E=\sqrt{Q^{2}}-M_{D^{0}}-M_{D^{* 0}}$.

From Eqs. (10) and (11) we rewrite the differential decay rate for Eq. (9) as

$$
\begin{aligned}
\frac{\mathrm{d} \Gamma_{B \rightarrow K F}}{\mathrm{~d} E} & =\frac{\Gamma_{B \rightarrow K X}\left(Q^{2}\right) \Gamma_{X \rightarrow F}\left(Q^{2}\right)}{2 \pi\left|\sqrt{Q^{2}}-P_{X}\right|^{2}} \\
& =\frac{\Gamma_{B \rightarrow K X}\left(Q^{2}\right) \Gamma_{X \rightarrow F}\left(Q^{2}\right)}{2 \pi\left|E-E_{X}+i \Gamma_{X} / 2\right|^{2}},
\end{aligned}
$$

with $E_{X}=M_{X}-M_{D^{0}}-M_{\bar{D}^{* 0}}$ the mass of the resonance from the $D^{0} \bar{D}^{* 0}$ threshold. ${ }^{4}$

\footnotetext{
4 We follow a different sign convention for $E_{X}$ compared to Ref. [3], so that here $E_{X}$ is negative for $M_{X}<M_{D^{0}}+M_{\bar{D}^{* 0}}$.
}

Next, let us assume that we describe the final-state interactions of the $D^{0} \bar{D}^{* 0}$ system in terms of a function $d(E)$ that gives account of the $X$ (3872) signal properly, which is represented in Eq. (12) by the propagator factor squared $1 /\left|E-E_{X}+i \Gamma_{X} / 2\right|^{2}$. This is strictly the case for a bound state or for an isolated resonance such that $\left|\Gamma_{X} / E_{X}\right| \ll 1$. For any other case (e.g. a pure virtual-state case) we make use of the analytical continuation of the expressions obtained. Then we can write $d(E)$ around this energy region as

$d(E) \simeq \frac{\alpha}{\left(E-P_{X}\right)}$,

with $\alpha$ the residue of $d(E)$ at the resonance pole. In this way,

$$
\frac{1}{\left|E-M_{X}+i \Gamma_{X} / 2\right|^{2}} \rightarrow \frac{|d(E)|^{2}}{|\alpha|^{2}},
$$

and we express Eq. (12) as

$$
\frac{\mathrm{d} \Gamma_{B \rightarrow K F}}{\mathrm{~d} E}=\Gamma_{B \rightarrow K X}\left(Q^{2}\right) \Gamma_{X \rightarrow F}\left(Q^{2}\right) \frac{|d(E)|^{2}}{2 \pi|\alpha|^{2}} .
$$

As in Refs. [1,3] it is convenient to introduce in Eq. (15) the product of the branching ratios for the decays $B \rightarrow K X$ and $X \rightarrow F, \mathcal{B}_{F}\left(Q^{2}\right)=\Gamma_{B \rightarrow K X}\left(Q^{2}\right) \Gamma_{X \rightarrow F}\left(Q^{2}\right) / \Gamma_{B} \Gamma_{X}$, with $\Gamma_{B}$ the total decay width of a $B$ meson. This equation then reads

$\frac{\mathrm{d} \Gamma_{B \rightarrow K F}}{\mathrm{~d} E}=\Gamma_{B} \mathcal{B}_{F}\left(Q^{2}\right) \frac{\Gamma_{X}|d(E)|^{2}}{2 \pi|\alpha|^{2}}$,

However, for a final system $F$ with a threshold relatively far away from the $D \bar{D}^{* 0}$ threshold compared to $\left|E_{X}\right|$, we can neglect the $Q^{2}$ dependence in $\mathcal{B}_{F}$. This criterion can also be applied to a $B \rightarrow K J / \psi \pi^{+} \pi^{-}$decay because of the rather large width of the $\rho$ around $150 \mathrm{MeV}$, which washes out the sharp threshold effect for this state if we neglected the $\rho$ width [20]. However, this is not the case for the $B \rightarrow K D^{0} \bar{D}^{* 0}$ decay measured by the BaBar [15] and Belle [17] Collaborations, which is discussed in the next section.

We also consider here the $J / \psi \pi^{+} \pi^{-}$event distributions from the inclusive $p \bar{p}$ collisions at $\sqrt{s}=1.65 \mathrm{TeV}$ measured by the CDF Collaboration [18]. The basic Feynman diagram now shown in Fig. 2. It is similar to Fig. 1 but changing the kaon $K$ by a set of undetected final particles that are denoted collectively as $F^{\prime}$, with $X(3872)$ decaying into a set of particles denoted by $F$ as above. Instead of Eq. (2) we

Fig. 2 Skeleton Feynman diagram for the $p \bar{p} \rightarrow F^{\prime} F$ scattering through the $X(3872)$ resonance

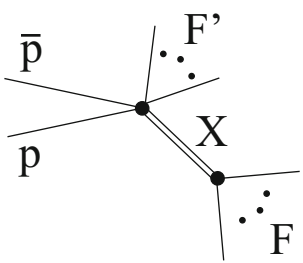


have now to calculate the cross section for $p \bar{p}$ to $F^{\prime} F$ that reads

$$
\begin{aligned}
\mathrm{d} \sigma_{p \bar{p} \rightarrow F^{\prime} F}= & \frac{1}{4 \sqrt{s}|p|} \int(2 \pi)^{4} \delta\left(P-P_{F^{\prime}}-Q\right) \mathrm{d} \mathcal{F}^{\prime} \mathrm{d} \mathcal{F} \\
& \times \frac{\left|\mathcal{V}_{L}\right|^{2}\left|\mathcal{V}_{X}\right|^{2}}{\left|Q^{2}-P_{X}^{2}\right|^{2}},
\end{aligned}
$$

where $|p|$ is the $\mathrm{CM}$ thee-momentum of the initial $p \bar{p}$ system and

$\mathrm{d} \mathcal{F}^{\prime}=\prod_{i=1}^{N_{F^{\prime}}} \frac{\mathrm{d}^{3} p_{i}}{(2 \pi)^{3} 2 E_{i}}$.

Splitting $\mathrm{d} \mathcal{F}$ as in Eq. (4), we rewrite Eq. (17) as

$$
\begin{aligned}
\mathrm{d} \sigma_{p \bar{p} \rightarrow F^{\prime} F}= & \int \frac{\mathrm{d} Q_{0}}{2 \pi} \mathrm{d} \mathfrak{f} 2 \sqrt{Q^{2}+\mathbf{Q}^{2}} \frac{\left|\mathcal{V}_{X}\right|^{2}}{\left|Q^{2}-P_{X}^{2}\right|^{2}} \\
& \times \frac{1}{4 \sqrt{s}|p|} \int(2 \pi)^{4} \delta\left(P-P_{F^{\prime}}-Q\right)\left|\mathcal{V}_{L}\right|^{2} \mathrm{~d} \mathcal{F}^{\prime} \\
& \times \frac{\mathrm{d}^{3} Q}{(2 \pi)^{3} 2 \sqrt{Q^{2}+\mathbf{Q}^{2}}} .
\end{aligned}
$$

Next, we perform the change of integration variable from $Q_{0}$ to $Q^{2}$, cf. Eq. (8), and after integrating over $\mathcal{F}^{\prime}$ and $\mathbf{Q}$ the factor on the right-hand side of the second line in the previous equation is $\sigma_{p \bar{p} \rightarrow X \text { All }}\left(Q^{2}\right)$, namely,

$$
\begin{aligned}
\sigma_{p \bar{p} \rightarrow X \mathrm{All}}\left(Q^{2}\right)= & \frac{1}{4 \sqrt{s}|p|} \int(2 \pi)^{4} \delta\left(P-P_{F^{\prime}}-Q\right)\left|\mathcal{V}_{L}\right|^{2} \mathrm{~d} \mathcal{F}^{\prime} \\
& \times \frac{\mathrm{d}^{3} Q}{(2 \pi)^{3} 2 \sqrt{Q^{2}+\mathbf{Q}^{2}}}
\end{aligned}
$$

Additionally, recalling the expression for $\Gamma_{X \rightarrow F}\left(Q^{2}\right)$ in Eq. (7), the Eq. (19) becomes

$\mathrm{d} \sigma_{p \bar{p} \rightarrow F^{\prime} F}=\sigma_{p \bar{p} \rightarrow X \operatorname{All}}\left(Q^{2}\right) \Gamma_{X \rightarrow F}\left(Q^{2}\right) \frac{2 \sqrt{Q^{2}}}{\left|Q^{2}-P_{X}^{2}\right|^{2}} \frac{\mathrm{d} Q^{2}}{2 \pi}$.

Approaching the inverse propagator as in Eq. (10) and employing $d(E)$ to take into account the FSI, we finally rewrite Eq. (21) as

$$
\frac{\mathrm{d} \sigma_{p \bar{p} \rightarrow F^{\prime} F}}{\mathrm{~d} E}=\sigma_{p \bar{p} \rightarrow X \operatorname{All}}\left(Q^{2}\right) \operatorname{Br}(X \rightarrow F)\left(Q^{2}\right) \frac{\Gamma_{X}|d(E)|^{2}}{2 \pi|\alpha|^{2}} .
$$

\section{$3 D^{0} \bar{D}^{* 0}$ partial-decay rate}

The $B \rightarrow K D^{0} \bar{D}^{* 0}$ decay rate measured be the reconstruction of $\bar{D}^{* 0}$ from the decay channels $D^{0} \bar{D}^{0} \pi^{0}$ and $D^{0} \bar{D}^{0} \gamma$ decays $[15,17]$ has a strong dependence on the $D^{0} \bar{D}^{* 0}$ invariant mass in the energy region of the $X(3872)$. One obvious reason is that $D^{0} \bar{D}^{* 0}$ is almost at threshold. Besides that, one also has the decay chain $B \rightarrow K X(3872)$,
$X(3872) \rightarrow D^{0} \bar{D}^{* 0}$ and finally $\bar{D}^{* 0} \rightarrow \bar{D}^{0} \pi^{0}$ or $\bar{D}^{0} \gamma$, so that the $\bar{D}^{* 0}$ Lorentzian has some overlapping with $X(3872)$ mass distribution that rapidly decreases for increasing energy if the latter lies below threshold. As a result, the width of the $\bar{D}^{* 0}$ has to be taken into account in the formalism from the start to study the decays of the $X(3872)$ through the $D^{0} \bar{D}^{* 0}$ intermediate state, particularly if this state manifests as a $D^{0} \bar{D}^{* 0}$ bound state. This point was stressed originally in Ref. [2].

A $D^{0} \bar{D}^{0} \pi^{0}$ event from the $B$ decays to $K X(3872)$ can be generated by either $B \rightarrow K X(3872), X(3872) \rightarrow \bar{D}^{0} D^{* 0}$, $D^{* 0} \rightarrow D^{0} \pi^{0}$ or $X(3872) \rightarrow D^{0} \bar{D}^{* 0}, \bar{D}^{* 0} \rightarrow \bar{D}^{0} \pi^{0}$. This is an interesting interference process for $X$ (3872) being mostly a $D^{0} \bar{D}^{* 0}$ molecule, as first discussed in Ref. [21]. This latter reference shows that the interference effects vanish for a zero binding energy while Ref. [3] elaborates that they can be neglected for $|E| \ll 2\left(M_{\pi^{0}} / M_{D^{0}}\right) \delta \simeq 1 \mathrm{MeV}$, with $\delta=M_{D^{* 0}}-M_{D^{0}}-M_{\pi^{0}} \simeq 7.2 \mathrm{MeV}$, the energy delivered in a $D^{* 0}$ decay at rest. For the case of $X(3872)$ with a nominal mass $E_{X}=-0.12 \pm 0.20 \mathrm{MeV}$ [19] (adding in quadrature the uncertainties in the masses of the $X(3872), D^{0}$ and $D^{* 0}$ given in the PDG [19]) this inequality is operative and one might expect some suppression of these interference effects. The latter were also worked out explicitly in Ref. [22] by considering the three-body $D^{0} \bar{D}^{0} \pi$ dynamics and it was shown there that for a binding energy of $0.5 \mathrm{MeV}$, the interference effects below the $D^{0} \bar{D}^{* 0}$ threshold at the peak of $X(3872)$ are sizable. This result is in agreement with the outcome of Ref. [21] for the decay width of $X(3872)$ to $D^{0} \bar{D}^{0} \pi^{0}$, which found that they are substantial already for binding energies $\left|E_{X}\right| \gtrsim 0.1 \mathrm{MeV}$. However, Ref. [22] derived that above the $D^{0} \bar{D}^{* 0}$ threshold they are very modest and for the case of a virtual state they are so in the whole energy range (both above and below threshold). Additionally, these interference effects are mostly proportional to the weight of the molecular $D^{0} \bar{D}^{* 0}$ weight of $X$ (3872) or compositeness, as explicitly shown by Voloshin in Ref. [21]. In turn, the interference contributions in the decay channel $D^{0} \bar{D}^{0} \gamma$ should be smaller because the three-momentum of $D^{0}$ from the decay $D^{* 0} \rightarrow D^{0} \gamma$ is significantly bigger than for $D^{0} \pi^{0}$, so that the overlapping with the wave function of $D^{0}$ in the $X(3872)$ is reduced, an argument borrowed from Ref. [21]. Based on these facts resulting from previous work $[3,21,22]$ and because we are mostly interested in our study in scenarios for the $X(3872)$ in which it is a double/triplet virtual state or it has a very small molecular component, we neglect in the following any interference effect in the $D \bar{D}^{0} \pi^{0}$ and $D \bar{D}^{0} \gamma$ decays. ${ }^{5}$ Then we first consider the diagonal processes and take for definiteness the

\footnotetext{
5 Mostly for comparison with less standard scenarios we also discuss the case of a pure molecular $X(3872)$ generated within the scattering length approximation [2,3]. In this case it is true that interference effects could be more important, around a $60 \%$ of the direct term at the resonance mass according to Refs. [21,22]. Nonetheless, we are interested
} 
chain of decays $B \rightarrow K X(3872), X(3872) \rightarrow \bar{D}^{0} D^{* 0}$ and finally $D^{* 0} \rightarrow D^{0} \pi^{0}$. The resulting decay width is denoted by $\gamma_{X \rightarrow \bar{D}^{0} D^{0} \pi^{0}}\left(Q^{2}\right)$, which should be multiplied by 2 to have the corresponding partial-decay width, $\Gamma_{X \rightarrow D^{0} \bar{D}^{0} \pi^{0}}\left(Q^{2}\right)$, in the limit in which we can neglect the aforementioned interference. Analogous steps would apply to the decay $B \rightarrow$ $K D^{0} \bar{D}^{0} \gamma$ through $X(3872)$.

Due to the closeness of $M_{X}$ and $M_{D^{0}}+M_{D^{* 0}}$ one cannot neglect the $Q^{2}$ dependence of $\Gamma_{X \rightarrow D^{0} \bar{D}^{0} \pi^{0}}\left(Q^{2}\right)$ in Eq. (15) as noticed at the end of Sect. 2. All the factors on the righthand side of Eq. (7) are Lorentz invariant and we evaluate it in the $X(3872)$ rest frame, where one finds the expression (with $F=D^{0} \bar{D}^{0} \pi^{0}$ )

$$
\begin{gathered}
\Gamma_{X \rightarrow \bar{D}^{0} D^{0} \pi^{0}}\left(Q^{2}\right)=\frac{\hat{\beta}^{2}}{2 \sqrt{Q^{2}}} \int(2 \pi)^{4} \delta\left(\mathbf{p}_{\bar{D}}+\mathbf{p}_{D}+\mathbf{p}_{\pi}\right) \\
\delta\left(\delta+E-\frac{\mathbf{p}_{\bar{D}}^{2}}{2 M_{\bar{D}^{0}}}-\frac{\mathbf{p}_{D}^{2}}{2 M_{D^{0}}}-\frac{\mathbf{p}_{\pi}^{2}}{2 M_{\pi^{0}}}\right) \frac{\mathrm{d}^{3} p_{D}}{(2 \pi)^{3} 2 E_{D}} \\
\quad \times \frac{\mathrm{d}^{3} p_{\bar{D}}}{(2 \pi)^{3} 2 E_{\bar{D}}} \frac{\mathrm{d}^{3} p_{\pi}}{(2 \pi)^{3} 2 E_{\pi}} \frac{p_{\pi}^{2}}{\left(2 M_{D^{* 0}}\right)^{2}\left|E+i \frac{\Gamma_{*}}{2}-\frac{\mathbf{p}_{\bar{D}}^{2}}{2 \mu}\right|^{2}} .
\end{gathered}
$$

Several points need be discussed concerning this equation. We have explicitly indicated the potentially most rapidly varying kinematical facts in the decay $X \rightarrow F$ that comprises the $D^{* 0}$ propagator and the $P$-wave character of $D^{* 0} \rightarrow D^{\pi^{0}}$, which implies the appearance of the momentum squared of the pion. ${ }^{6}$ In Eq. (23) we have indicated by $\hat{\beta}^{2}$ a coupling constant squared, by $\mu$ the $\bar{D}^{0} D^{* 0}$ reduced mass $\left(\mu=M_{D^{0}} M_{D^{* 0}} /\left(M_{D^{0}}+M_{D^{* 0}}\right)\right.$ and by $\Gamma_{*}$ the $D^{* 0}$ width. We have used the non-relativistic reduction for the energies of $D^{0}, \bar{D}^{0}$ and $\pi^{0}$, as mass plus kinetic energy, in the Dirac delta function for the conservation of energy. This is also quite valid for the pion because $\delta \ll M_{\pi^{0}}$. The nonrelativistic expression is used for the $D^{* 0}$ propagator as well. Let us see how it emerges from its relativistic form:

Footnote 5 continued

in the $D^{0} \bar{D}^{* 0}$ production above its threshold for which the effect at the peak of $X(3872)$ is reduced by the remarkably narrow Lorentzian associated with the $D^{* 0}$ resonance for physical energies $\left(\mathcal{E}^{\prime}>0\right)$, cf. Eq. (25). In addition we also have the contribution from the $D^{0} \bar{D}^{* 0}$ production above threshold, which is of similar size as the former for the $X(3872)$ signal region in the pure molecular bound-state case, as we have checked. For this case we then expect to do an error estimated to be smaller than a $30 \%$, already of similar size as the experimental error, which can easily be accounted for by a renormalization about the same amount of the normalization constant multiplying the signal contribution.

6 At the end $p_{\pi} \simeq \sqrt{2 M_{\pi^{0} \delta}}$ because $\delta \gg\left|E_{X}\right|$, and it could be re-absorbed in $\hat{\beta}^{2}$ of Eq. (23).

$$
\begin{aligned}
& p_{D^{*}}^{2}-\left(M_{D^{* 0}}-i \frac{\Gamma_{*}}{2}\right)^{2} \\
& =\left(M_{D^{* 0}}+E-\frac{\mathbf{p}_{\bar{D}}^{2}}{2 M_{\overline{D^{0}}}}\right)^{2}-\mathbf{p}_{\bar{D}}^{2}-\left(M_{D^{* 0}}-i \frac{\Gamma_{*}}{2}\right)^{2},
\end{aligned}
$$

where we have employed the non-relativistic expression for the energy of $\bar{D}^{0}$ and that in the rest frame of the $X(3872)$, $\mathbf{p}_{\bar{D}}+\mathbf{p}_{D^{*}}=0$. Neglecting quadratic terms in $E$, kinetic energies and $\Gamma_{*}$ we are lead to the expression for the $D^{* 0}$ propagator used in Eq. (23). Next, we insert in this equation the integral identity

$$
\begin{aligned}
1= & \int(2 \pi)^{4} \delta\left(\mathbf{p}_{D^{*}}-\mathbf{p}_{D}-\mathbf{p}_{\pi}\right) \delta\left(\delta+\mathcal{E}+\frac{\mathbf{p}_{D^{*}}^{2}}{2 M_{D^{* 0}}}\right. \\
& \left.-\frac{\mathbf{p}_{D}^{2}}{2 M_{D^{0}}}-\frac{\mathbf{p}_{\pi}^{2}}{2 M_{\pi^{0}}}\right) \frac{\mathrm{d}^{3} p_{D^{*}}}{(2 \pi)^{3}} \frac{\mathrm{d} \mathcal{E}}{2 \pi},
\end{aligned}
$$

which corresponds to an intermediate $D^{* 0}$ with threemomentum $\mathbf{p}_{D^{*}}$ and energy $E_{D^{*}}=M_{D^{* 0}}+\mathbf{p}_{D^{*}}^{2} / 2 M_{D^{* 0}}+\mathcal{E}$. In this way we are explicitly extracting the phase space factor corresponding to the final $D^{0} \pi^{0}$ in the $D^{* 0}$ decay, similarly as done above in Eqs. (4) and (5) for the $X$ (3872) resonance and the subsystem $F$. We use this result to rewrite Eq. (23) as

$$
\begin{aligned}
& \gamma_{X \rightarrow \bar{D}^{0} D^{0} \pi^{0}}\left(Q^{2}\right)=\frac{\hat{\beta}_{1}^{2}}{2 \sqrt{Q^{2}}} \int(2 \pi)^{4} \delta\left(\mathbf{p}_{\bar{D}}+\mathbf{p}_{D^{*}}\right) \\
& \delta\left(E-\mathcal{E}-\frac{\mathbf{p}_{\bar{D}}^{2}}{2 \mu}\right) \frac{\mathrm{d}^{3} p_{\bar{D}}}{(2 \pi)^{3} 2 E_{\bar{D}}} \frac{\mathrm{d}^{3} p_{D^{*}}}{(2 \pi)^{3}} \frac{\mathrm{d} \mathcal{E}}{2 \pi} \\
& \times \frac{1}{2 M_{D^{* 0}}\left|E+i \frac{\Gamma_{*}}{2}-\frac{\mathbf{p}_{\bar{D}}^{2}}{2 \mu}\right|^{2}} \\
& \times \frac{\hat{\beta}_{2}}{2 M_{D^{* 0}}} \int(2 \pi)^{4} \delta\left(\mathbf{p}_{D^{*}}-\mathbf{p}_{D}-\mathbf{p}_{\pi}\right) \\
& \delta\left(\delta+\mathcal{E}+\frac{\mathbf{p}_{\bar{D}}^{2}}{2 M_{D^{* 0}}}-\frac{\mathbf{p}_{D}^{2}}{2 M_{D^{0}}}-\frac{\mathbf{p}_{\pi}^{2}}{2 M_{\pi^{0}}}\right) p_{\pi}^{2} \\
& \quad \times \frac{\mathrm{d}^{3} p_{D}}{(2 \pi)^{3} 2 E_{D}} \frac{\mathrm{d}^{3} p_{\pi}}{(2 \pi)^{3} 2 E_{\pi}},
\end{aligned}
$$

where we have split $\hat{\beta}=\hat{\beta}_{1} \hat{\beta}_{2}$, such that the term on the right-hand side of the last line in the previous equation can be identified with the partial-decay width $D^{* 0} \rightarrow D^{0} \pi^{0}$ at rest, which we denote $\Gamma_{D^{* 0} \rightarrow D^{0} \pi^{0}}$. As in Eq. (7) we see that this partial-decay width should be strictly evaluated at the corresponding $D^{* 0}$ invariant mass. However, since $X$ (3872) is so close to the $D^{0} \bar{D}^{* 0}$ threshold and $\sqrt{Q^{2}} \simeq M_{X}^{2}$ we can simply take the invariant mass of $D^{* 0}$ to be equal to $M_{D^{* 0}}$, which furthermore has a tiny width.

Regarding the factor on the right-hand side of the first line in Eq. (26) the integration over $\mathbf{p}_{D^{*}}$ and $\mathbf{p}_{\bar{D}}$ are straightforward, and then we are left with 
$\frac{\hat{\beta}_{1}^{2} \mu^{\frac{3}{2}}}{4 \pi \sqrt{2 Q^{2}} M_{D^{* 0}}} \int_{-\infty}^{E} \frac{\mathrm{d} \mathcal{E}}{2 \pi} \frac{\sqrt{E-\mathcal{E}}}{E_{\bar{D}}\left(\mathcal{E}^{2}+\frac{\Gamma_{*}^{2}}{4}\right)}$,

where $\left|\mathbf{p}_{\bar{D}}\right|=\sqrt{2 \mu(E-\mathcal{E})}$. The integration in the previous equation is a convergent one within a range that gives rise to tiny kinetic energies compared to $M_{\bar{D}}$ in Eq. (27). Then we can just keep the dominant $\left|\mathbf{p}_{\bar{D}}\right|$ dependence that stems from the factor $\sqrt{E-\mathcal{E}}$ in the numerator of the integrand and replace $E_{\bar{D}}$ by $M_{D^{0}}$. In terms of the new integration variable $\mathcal{E}^{\prime}$, defined as

$\mathcal{E}^{\prime}=E-\mathcal{E}$,

the integral is now

$$
\begin{aligned}
& \frac{\hat{\beta}_{1}^{2} \mu^{\frac{3}{2}}}{4 \pi \sqrt{2 Q^{2}} M_{D^{* 0}} M_{D^{0}}} \int_{0}^{+\infty} \frac{\mathrm{d} \mathcal{E}^{\prime}}{2 \pi} \frac{\sqrt{\mathcal{E}^{\prime}}}{\left(\mathcal{E}^{\prime}-E\right)^{2}+\frac{\Gamma_{*}^{2}}{4}} \\
& =\frac{\hat{\beta}_{1}^{2} \mu^{\frac{3}{2}}}{8 \pi \sqrt{Q^{2}} M_{D^{* 0} M_{D^{0} \Gamma_{*}}} \sqrt{E+\sqrt{E^{2}+\Gamma_{*}^{2} / 4}}} \\
& =\frac{\Gamma_{X \rightarrow \bar{D}^{0} D^{* 0}}}{\Gamma_{*}} \frac{\sqrt{E+\sqrt{E^{2}+\Gamma_{*}^{2} / 4}}}{\sqrt{E_{X}+\sqrt{E_{X}^{2}+\Gamma_{*}^{2} / 4}}},
\end{aligned}
$$

with

$$
\Gamma_{X \rightarrow \bar{D}^{0} D^{* 0}}=\frac{\hat{\beta}_{1}^{2} \mu^{\frac{3}{2}} \sqrt{E_{X}+\sqrt{E_{X}^{2}+\Gamma_{*}^{2} / 4}}}{8 \pi \sqrt{Q^{2}} M_{D^{* 0}} M_{D^{0}}} .
$$

To get the total partial-decay width of $X(3872)$ into $D^{0} \bar{D}^{0} \pi^{0}$ we still have to multiply Eq. (29) by 2 because of the two mechanisms involved, $X(3872) \rightarrow \bar{D}^{0} D^{* 0}$ (the one explicitly analyzed) and $X(3872) \rightarrow D^{0} \bar{D}^{* 0}$. As argued above we are neglecting interference effects. Then we end from Eqs. (15), (26) and (29) with the following expression for the partial-decay rate $B \rightarrow K D^{0} \bar{D}^{0} \pi^{0}$ :

$\frac{\mathrm{d} \Gamma_{B \rightarrow K D^{0} \bar{D}^{0} \pi^{0}}}{\mathrm{~d} E}=\mathcal{B}_{D \pi} \Gamma_{B} \Gamma_{X} \frac{\sqrt{E+\sqrt{E^{2}+\Gamma_{*}^{2} / 4}}}{\sqrt{E_{X}+\sqrt{E_{X}^{2}+\Gamma_{*}^{2} / 4}}} \frac{|d(E)|^{2}}{2 \pi|\alpha|^{2}}$,

where $\mathcal{B}_{D \pi}=\Gamma_{B \rightarrow K X} \Gamma_{X \rightarrow D^{0} \bar{D}^{* 0}} \Gamma_{D^{* 0} \rightarrow D^{0} \pi^{0}} / \Gamma_{B} \Gamma_{X} \Gamma_{*}$. This equation was already derived in Ref. [3] and its main characteristic energy dependence found before in Ref. [2].

However, what is measured experimentally is the $\bar{D}^{0} D^{* 0}$ invariant mass $[15,17]$, which is given by $\left|\mathbf{p}_{\bar{D}}\right|^{2} / 2 \mu$ when measured with respect to the $D^{0} \bar{D}^{* 0}$ threshold in the $X$ (3872) rest frame. As indicated above because of the energy conservation Dirac delta function in the first line of Eq. (26) this quantity is equal to $\mathcal{E}^{\prime}$. Thus, instead of the differential rate $\mathrm{d} \Gamma_{B \rightarrow K D^{0} \bar{D}^{0} \pi^{0}} / \mathrm{d} E$ we should compare the experimental data with $\mathrm{d} \Gamma_{B \rightarrow K D^{0} \bar{D}^{0} \pi^{0}} / \mathrm{d} \mathcal{E}^{\prime}$. The latter can be calculated from Eq. (29) by removing the integration in $\mathcal{E}^{\prime}$, and replacing $\frac{\hat{\beta}_{1}^{2} \mu^{\frac{3}{2}}}{8 \pi \sqrt{Q^{2}} M_{D^{* 0}} M_{D^{0}} \Gamma_{*}}$ in terms of $\Gamma_{X \rightarrow \bar{D}^{0} D^{* 0}}$, cf. Eq. (30). The result is multiplied by $\Gamma_{D^{*} \rightarrow D^{0} \pi^{0}}$, which is present in Eq. (26), and by 2 because of the two ways of decay involved. This is then placed in Eq. (15) instead of $\Gamma_{X \rightarrow F}$, which is then integrated with respect to $E$. We end with,

$$
\begin{aligned}
\frac{\mathrm{d} \Gamma_{B \rightarrow K D^{0} \bar{D}^{0} \pi^{0}}}{\mathrm{~d} \mathcal{E}^{\prime}}= & \frac{\Gamma_{B} \mathcal{B}_{D \pi} \sqrt{\mathcal{E}^{\prime}}}{\sqrt{2} \pi \sqrt{E_{X}+\sqrt{E_{X}^{2}+\Gamma_{*}^{2} / 4}}} \int_{-\infty}^{+\infty} \mathrm{d} E \\
& \times \frac{\Gamma_{*}}{\left(\mathcal{E}^{\prime}-E\right)^{2}+\frac{\Gamma_{*}^{2}}{4}} \frac{\Gamma_{X}|d(E)|^{2}}{2 \pi|\alpha|^{2}}
\end{aligned}
$$

This expression coincides with the one already deduced in Ref. [3]. However, our derivation proceeds in a more straightforward manner by having split the $D^{0} \bar{D}^{0} \pi^{0}$ phase space factor in two terms of lower dimensionality [19], attached to the decays $X(3872) \rightarrow \bar{D}^{0} D^{* 0}$ and $D^{* 0} \rightarrow D^{0} \pi^{0}$, employing Eq. (25). In this way the variable $\mathcal{E}^{\prime}$ enters directly into the formulas.

Analogous steps can be followed to derive the corresponding expression for $\mathrm{d} \Gamma_{B^{+} \rightarrow K^{+} D^{0} \bar{D}^{0} \gamma} / \mathrm{d} \mathcal{E}^{\prime}$ and when summed to Eq. (32) we have

$$
\begin{aligned}
\frac{\mathrm{d} \Gamma_{B^{+} \rightarrow K^{+} D^{0} \bar{D}^{* 0}}}{\mathrm{~d} \mathcal{E}^{\prime}}= & \frac{\Gamma_{B^{+}} \mathcal{B}_{D} \sqrt{\mathcal{E}^{\prime}}}{\sqrt{2} \pi \sqrt{E_{X}+\sqrt{E_{X}^{2}+\Gamma_{*}^{2} / 4}}} \int_{-\infty}^{+\infty} \mathrm{d} E \\
& \times \frac{\Gamma_{*}}{\left(\mathcal{E}^{\prime}-E\right)^{2}+\frac{\Gamma_{*}^{2}}{4}} \frac{\Gamma_{X}|d(E)|^{2}}{2 \pi|\alpha|^{2}}
\end{aligned}
$$

with

$$
\begin{aligned}
\mathcal{B}_{D} & =\frac{\Gamma_{B \rightarrow K X} \Gamma_{X \rightarrow D^{0} \bar{D}^{* 0}}\left(\Gamma_{D^{* 0} \rightarrow D^{0} \pi^{0}}+\Gamma_{D^{* 0} \rightarrow D^{0} \gamma}\right)}{\Gamma_{B} \Gamma_{X} \Gamma_{*}} \\
& =\frac{\Gamma_{B \rightarrow K X} \Gamma_{X \rightarrow D^{0} \bar{D}^{* 0}}}{\Gamma_{B} \Gamma_{X}} .
\end{aligned}
$$

The last equality follows by taking a $100 \%$ branching ratio for the partial-decay width of a $D^{* 0}$ into $D^{0} \pi^{0}$ and $D^{0} \gamma$ [19].

\section{Final-state interactions}

As discussed above in the Introduction the applicability of the ERE (and hence of a Flatté parameterization as well) to study near-threshold resonances, their properties and nature [1$6,10]$, could be severely limited by the presence of a nearby zero in the partial-wave amplitude.

This interplay between a resonance and a close zero indeed recalls the situation in the presence of the Adler zero required by chiral symmetry in the isoscalar scalar pion-pion $(\pi \pi)$ 
scattering and the associated $\sigma$ or $f_{0}(500)$ resonance. The presence of this zero distorts strongly the $f_{0}(500)$ resonance signal in $\pi \pi$ scattering while for several production processes this zero is not required by any fundamental reason and it does not show up. This is why the $f_{0}(500)$ resonance could be clearly observed experimentally with high statistics significance in $D \rightarrow \pi \pi \pi$ decays [23], where the $S$-wave $\pi \pi$ final-state interactions are mostly sensitive to the pion scalar form factor which is free of any low-energy zero; see e.g. Refs. [24-28] for related discussions.

Regarding $X(3872)$ there are data on event distributions involving $J / \psi[14,16,18,29]$ that show a clean eventdistribution signal for this resonance without any distortion caused by a zero. However, this does not exclude that a zero could be relevant for the near-threshold $D^{0} \bar{D}^{* 0}$ scattering, as it indeed happens for the $f_{0}(500)$ case. Of course, the situation is not completely analogous because here $X(3872)$ is almost on top of the $D^{0} \bar{D}^{* 0}$ threshold and it has a very small width, while $f_{0}(500)$ is wide and one does use the ERE to study it because it is too far away from the $\pi \pi$ threshold. This implies that a CDD pole in the present problem on $D^{0} \bar{D}^{* 0}$ scattering must be really close to its threshold so as to spoil the applicability of the ERE.

In this way, instead of using the ERE as in Refs. [1-6] we employ another more general parameterization that comprises the ERE up to the effective-range contribution (indeed up to the next shape parameter) for some limiting case but at the same time it is also valid even in the presence of a nearthreshold CDD pole. This parameterization can be deduced by making use of the $N / D$ method as done in Ref. [30], whose non-relativistic reduction is given in Ref. [31]. The point is to perform a dispersion relation of the inverse of the $D^{0} \bar{D}^{* 0} S$-wave $t(E)$, which along the unitarity cut fulfills the well-known unitarity relation

$\operatorname{Im} t(E)^{-1}=-k(E), \quad E \geq 0$,

where $E$ is the center of mass (CM) energy of the system, cf. Eq. (11), and $k(E)$ is the CM three-momentum given by its non-relativistic reduction $k(E)=\sqrt{2 \mu E}$. Next, we neglect crossed-channel dynamics based on the fact that the scale $\Lambda$ associated with the massless one-pion exchange potential, as worked out in Refs. [12,32], is $\Lambda=4 \pi f_{\pi}^{2} / \mu g^{2} \sim 350 \mathrm{MeV}$ ( $f_{\pi}=92.4 \mathrm{MeV}$ and $g \simeq 0.6$ ), which is much bigger than the $D^{0} \bar{D}^{* 0}$ three-momentum $(\lesssim 30 \mathrm{MeV})$ in the region of the $X(3872)$. In this estimate one takes into account that the denominator in the exchange of a $\pi^{0}$ of momentum $\mathbf{q}$ between $D^{* 0}$ and $D^{0}$ is $\mathbf{q}^{2}+M_{\pi^{0}}^{2}-\left(M_{D^{* 0}}-M_{D^{0}}\right)^{2}$ and that $\left(\left(M_{D^{* 0}}-M_{D^{0}}\right)^{2}-M_{\pi^{0}}^{2}\right) / M_{\pi^{0}}^{2} \simeq 0.1 \ll 1$ because $M_{D^{* 0}}-M_{D^{0}}$ is larger than $M_{\pi^{0}}$ by only $7.2 \mathrm{MeV}$ [33]. It is then appropriate to write down a dispersion relation for $t(E)^{-1}$ with at least one necessary subtraction employing the

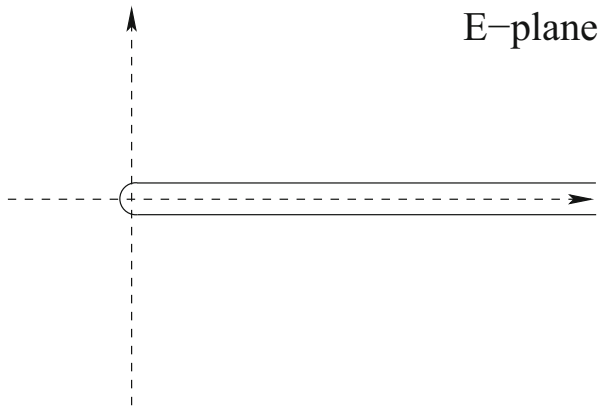

Fig. 3 Integration contour used for the dispersion relation of $t(E)^{-1}$ giving rise to Eq. (36). The contour is closed by a circle centered at the origin and of infinite radius

integration contour of Fig. 3. Then allowing for the presence of a pole of $t(E)^{-1}$ we then obtain

$t(E)=\left(\frac{\lambda}{E-M_{\mathrm{CDD}}}+\beta-i k(E)\right)^{-1}$,

with $M_{\mathrm{CDD}}$ the position of the CDD pole measured with respect to the $D^{0} \bar{D}^{* 0}$ threshold. Notice that this is a pole in $t(E)^{-1}$ and then a zero of $t(E)$ at $E=M_{\mathrm{CDD}}$.

Since the finite width effects of $D^{* 0}$ could be important as argued in Sect. 3, the CM three-momentum $k(E)$ is finally calculated according to the expression

$k(E)=\sqrt{2 \mu\left(E+i \frac{\Gamma_{*}}{2}\right)}$.

For definiteness the three-momentum $k(E)$ is always defined in the first Riemann sheet (RS), so that the phase of the radicand is taken between 0 and $2 \pi$. Here an analytical extrapolation in the mass of the $D^{* 0}$ resonance until its pole position $M_{D^{* 0}}-i \Gamma_{*} / 2$ has been performed, as also done e.g. in Refs. $[3,34,35]$. By considering explicitly the three-body channel $D^{0} \bar{D}^{0} \pi^{0}$ in a coupled-channel formalism, Ref. [22] found that Eq. (37) is appropriate because of the smallness of the $P$-wave $D^{* 0}$ width into $D^{0} \pi^{0}$, which implies that $\Gamma_{*} / 2 \delta=4.510^{-3} \ll 1$. In Eq. (36) the constant $\beta$ for elastic $D \bar{D}^{* 0}$ scattering is real but it becomes complex, with negative imaginary part, when taking into account inelasticities from other channels, such as $J / \psi \pi^{+} \pi^{-}, J / \psi \pi^{+} \pi^{-} \pi^{0}$, etc. [1-3]. We finally fix this possible imaginary part in $\beta$ to zero because, as already noticed in Ref. [3], one can reproduce data equally well, as we have also checked.

An ERE of $t(E)$ given in Eq. (36) is valid in the $k^{2}$ complex plane with a radius of convergence coincident with $2 \mu M_{\mathrm{CDD}}$. Notice that a zero of $t(E)$ near threshold implies that $k \cot \delta=\infty$ at this point and then it becomes singular. As a result, its $k^{2}$ expansion does not converge and the ERE becomes meaningless for practical applications since its radius of convergence is too small. In such a case, one 
must consider the full expression for $t(E)$ in Eq. (36) and not its ERE, which reads

$$
\begin{aligned}
k \cot \delta & =\frac{\lambda}{k^{2} / 2 \mu-M_{\mathrm{CDD}}}+\beta \\
& =-\frac{\lambda}{M_{\mathrm{CDD}}}+\beta-\frac{k^{2} \lambda}{2 \mu M_{\mathrm{CDD}}^{2}}+\cdots \\
& =-\frac{1}{a}+\frac{1}{2} r k^{2}+\cdots
\end{aligned}
$$

here the ellipsis indicate higher powers of $k^{2}$. This expansion can reproduce any values of the scattering length and effective range (as well as of the next shape parameter $v_{2}$ ) and we obtain the expressions ${ }^{7}$

$$
\begin{aligned}
\frac{1}{a} & =\frac{\lambda}{M_{\mathrm{CDD}}}-\beta, \\
r & =-\frac{\lambda}{\mu M_{\mathrm{CDD}}^{2}} .
\end{aligned}
$$

It is then clear that in order to generate a large absolute value for $a$, one needs a strong cancellation between $\lambda / M_{\mathrm{CDD}}$ and $\beta$ unless both of them are separately small. But in order to have a small magnitude of $|a|$ and a large one for $|r|$, one would naturally expect that $M_{\mathrm{CDD}} \rightarrow 0$, though the explicit value of $\lambda$ plays also an important role. Equation (39) clearly shows why the ERE could fail to converge even for very small values of $|k|^{2}$ as long as $M_{\mathrm{CDD}} \rightarrow 0$.

In the limit $M_{\mathrm{CDD}} \rightarrow \infty$ with $\lambda / M_{\mathrm{CDD}}$ fixed, the parameterization in Eq. (36) for $t(E)$ reduces to the function $f(E)$ used in Refs. [2,3]

$$
\begin{aligned}
t(E) & \underset{M_{\mathrm{CDD}} \rightarrow \infty}{\longrightarrow} f(E)=\frac{1}{-\frac{\lambda}{M_{\mathrm{CDD}}}+\beta-i k(E)} \\
& \lambda / M_{\mathrm{CDD}}=c t . \\
\equiv & \frac{1}{-\gamma-i k(E)}
\end{aligned}
$$

where $\gamma=1 / a$ is the inverse of the scattering length, using the notation of Ref. [3]. The function $f(E)$ has a bound (virtual) state pole for positive (negative) $\gamma$.

While the near-threshold energy dependence of $f(E)$ is dominated by the threshold branch-point singularity and a possible low-energy pole associated with a bound or virtual state, this is not necessarily the case for $t(E)$ as long as $M_{\mathrm{CDD}}$ is small enough. In such a case one has to explicitly remove the CDD pole from $t(E)$ by dividing it by $E-M_{\mathrm{CDD}}$. In this way, we end with the new function $d(E)$, already introduced in Sect. 2 just before Eq. (13), which is then defined as

$d(E)=\frac{1}{1+\frac{E-M_{\mathrm{CDD}}}{\lambda}(\beta-i k)}$,

\footnotetext{
7 Note the different sign convention for the scattering length here as compared with Ref. [31].
}

such that its low-energy behavior is qualitatively driven by the same facts mentioned for $f(E)$. This is also the function that in general terms drives final-state interactions (FSI) when the scattering partial wave is given by $t(E)$ in Eq. (36). A detailed account of it can be found in Ref. [31], although Ref. [26,27] could be more accessible depending on the reader's taste and education.

Next, we explicitly calculate the residue $\alpha$ for $d(E)$ needed to work out the decay rates in Eqs. (16) and (33) and the differential cross section of Eq. (22). This can be straightforwardly determined by moving to the pole position as defined in Eq. (13). Thus, it results

$\alpha=\frac{\xi}{1+\xi \eta}$

with

$\xi=-i \frac{k_{P}\left(\beta-i k_{P}\right)}{\mu}$,

$\eta=\frac{\beta-i k_{P}}{\lambda}$.

The three-momentum $k_{P}$ is evaluated at the pole position $E_{P}$ in the energy plane,

$k_{P}=\sqrt{2 \mu\left(E_{P}+i \frac{\Gamma_{*}}{2}\right)}$,

such that the phase of the radicand is between $[0,2 \pi$ [ for a bound-state pole in the first RS, while for a pole in the second RS, a virtual-state one, the phase is between $[2 \pi, 4 \pi$ [ and the sign of $k_{P}$ is reversed compared to its value in the first RS.

The constant $\alpha$, in the case of using the function $f(E)$ in Eq. (40) for the decay rates in Eqs. (16) and (33), is defined analogously as the residue of $f(E)$ at the pole position $P_{X}$. The function $f(E)$ has a different normalization compared to $d(E)$, and $\alpha$ is then given by

$\alpha=\frac{i k_{P}}{\mu}=-\frac{\gamma}{\mu}$.

Here we have taken into account that $k_{P}=i \gamma$ for the $f(E)$ parameterization.

The limit of decoupling a bare resonance from a continuum channel, like $D^{0} \bar{D}^{* 0}$, requires the presence of a zero to remove the pole of the resonance from $t(E)$. This simple argument shows that CDD poles and weakly coupled bare resonances are typically related. In this respect, we consider the resulting $t(E)$ obtained in Ref. [11] by considering the interplay between mesonic and quark degrees of freedom, and that results by considering the exchange of a bare resonance together with direct scattering terms in the mesonic channel at the level of the scattering length approximation. In the following discussions until the end of this section the 
zero width limit of $D^{* 0}$ should be understood in $k(E)$. The resulting $S$-wave amplitude from Ref. [11] is ${ }^{8}$

$t(E)=-\frac{1}{4 \pi^{2} \mu} \frac{E-E_{f}+\frac{1}{2} g_{f} \gamma_{V}}{\left(E-E_{f}\right)\left(\gamma_{V}+i k\right)+i \frac{1}{2} g_{f} \gamma_{V} k}$

Here, $a_{V}$ is the scattering length for the direct $D^{0} \bar{D}^{* 0}$ scattering (referred as potential scattering in Ref. [11]), $\gamma_{V}=1 / a_{V}$, $g_{f}$ is the coupling squared between the bare resonance and the mesonic channels, while $E_{f}$ is the mass of the bare resonance in the decoupling limit $g_{f} \rightarrow 0$. By comparing $t(E)$ in Eq. (46) with our expression above Eq. (36) one has the following relation between the parameters:

$$
\begin{array}{r}
\beta=-\gamma_{V}, \\
\lambda=\frac{1}{2} g_{f} \gamma_{V}^{2}, \\
M_{\mathrm{CDD}}=E_{f}-\frac{1}{2} g_{f} \gamma_{V},
\end{array}
$$

which shows that the results of Ref. [11] are a particular case of ours, since it is always possible to adjust $\lambda, M_{\mathrm{CDD}}$ and $\beta$ in terms of $g_{f}, E_{f}$ and $\gamma_{V}$. However, the reverse is not true because $g_{f} \geq 0$ [11], which implies that $\lambda$ is restricted to be positive as well, while the residue of the CDD pole can have a priori any sign. This difference is also important phenomenologically because, while our parameterization for $t(E)$ can give rise to values of the effective range with any sign, Ref. [11] generates only negative ones, cf. Eq. (39). Equation (47) explicitly shows the above remark that $E_{f} \rightarrow M_{\mathrm{CDD}}$ in the decoupling limit, $g_{f} \rightarrow 0$, with both $g_{f}$ and $M_{\mathrm{CDD}}-E_{f}$ being proportional to the residue of the CDD pole. It is also interesting to notice that $\beta$ corresponds to the minus the inverse of the potential scattering length $a_{V}$. The language of the exchange of a bare resonance and direct $D^{0} \bar{D}^{* 0}$ scattering could be more intuitive in some aspects than the direct use of $S$-matrix theory, employed to obtain Eq. (36), so that we will make contact with the former when discussing our findings. The formalism of Ref. [11] was extended to coupled channels in Ref. [13], and the inclusion of inelastic channels was also addressed more recently in Ref. [36,37].

The scattering length approximation for the $D^{0} \bar{D}^{* 0} S$ wave of Refs. [2,38] was further generalized in Ref. [12] to include as well the exchange of one bare resonance together with the explicit coupling between the channels $D^{0} \bar{D}^{* 0}$ and $D^{+} \bar{D}^{*-}$. The expression obtained in Ref. [12] for the elastic $D^{0} \bar{D}^{* 0} S$-wave amplitude is

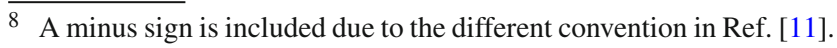

$$
\begin{aligned}
t_{11}(E)= & \frac{\left(-\gamma_{1}-\gamma_{0}+2 \kappa_{2}\right)\left(E-v+g^{2} \gamma_{0}\right)+g^{2} \gamma_{0}^{2}}{D(E)}, \\
D(E)= & \left(2 \gamma_{1} \gamma_{0}-\left(\gamma_{0}+\gamma_{1}\right)\left(\kappa_{2}-i k\right)-i 2 \kappa_{2} k\right) \\
& \times\left(E-v+g^{2} \gamma_{0}\right)+g^{2} \gamma_{0}^{2}\left(-2 \gamma_{1}+\kappa_{2}-i k\right) .
\end{aligned}
$$

Here, $\kappa_{2}=\sqrt{2 \mu\left(\Delta-E-i 0^{+}\right)}$, where $\Delta$ is the difference between the thresholds of $D^{-} D^{*+}$ and $\bar{D}^{0} D^{* 0}$. Additionally, $\gamma_{0,1}$ are the isoscalar and isovector scattering lengths in the limit of decoupling the bare state with the continuum channels and $g$ is the coupling among them. The parameter $v$ is the mass of the bare state measured with respect to the lightest threshold. To match Eq. (48) in the near-threshold region with the expression for $t(E)$ in Eq. (36) we rewrite the former as

$$
\begin{aligned}
& t(E)=(-i k+ \\
& \left.\frac{\left(E-v+g^{2} \gamma_{0}^{2}\right)\left(2 \gamma_{0} \gamma_{1}-\left(\gamma_{0}+\gamma_{1}\right) \kappa_{2}\right)-g^{2} \gamma_{0}^{2}\left(2 \gamma_{1}-\kappa_{2}\right)}{\left(E-v+g^{2} \gamma_{0}^{2}\right)\left(-\gamma_{1}-\gamma_{0}+2 \kappa_{2}\right)+g^{2} \gamma_{0}^{2}}\right)^{-1},
\end{aligned}
$$

which explicitly shows the correct form to fulfill elastic unitarity below the $D^{+} D^{*-}$ threshold, so that the term involving the product $\kappa k$ in Eq. (48) has disappeared. Restricting ourselves to our region of interest, $|E| \ll \Delta$, we can perform a Taylor expansion of $\kappa_{2}$ around $E=0$ and keep only its leading term $\kappa_{2} \rightarrow \hat{\kappa}_{2}=\sqrt{2 \mu \Delta}$, so that all the energy dependence of $t(E)^{-1}$ is dominated by the CDD pole and the right-hand cut for elastic scattering, as in our derivation of Eq. (36). ${ }^{9}$ The explicit expressions of $\lambda, \beta$ and $M_{\mathrm{CDD}}$ as a function of the parameters $\gamma_{0,1}, v, g^{2}$ and $v$ in Eq. (49) are

$$
\begin{array}{r}
\lambda=\frac{2 g^{2} \gamma_{0}^{2}\left(\gamma_{1}-\hat{\kappa}_{2}\right)^{2}}{\left(\gamma_{0}+\gamma_{1}-2 \hat{\kappa}_{2}\right)^{2}} \\
M_{Z}=\nu-\frac{g^{2} \gamma_{0}\left(\gamma_{1}-2 \hat{\kappa}_{2}\right)}{\gamma_{0}+\gamma_{1}-2 \hat{\kappa}_{2}} \\
\beta=\frac{-2 \gamma_{0} \gamma_{1}+\left(\gamma_{0}+\gamma_{1}\right) \hat{\kappa}_{2}}{\gamma_{0}+\gamma_{1}-2 \hat{\kappa}_{2}} .
\end{array}
$$

The same comment as made above, concerning the nonfully equivalence between our parameterization and the one of Ref. [11], is also in order here regarding Eq. (48). The point is that the latter implies again from Eq. (50) that $\lambda \geq 0$ while the residue of a CDD pole can have any sign.

\footnotetext{
9 If higher orders are kept in the Taylor expansion of $\kappa_{2}$ around $E=$ 0 then the matching would require one to include more CDD poles (with contributions suppressed by powers of $E / \Delta$ ); see Ref. [30] for details. Another option is to follow the coupled-channel formalism there developed as well, particularly when considering a wider energy range.
} 


\section{Formulas for the event distribution}

The combination $|d(E)|^{2} \Gamma_{X} / 2 \pi|\alpha|^{2}$ in Eqs. (16), (22) and (33) corresponds to the normalized standard non-relativistic mass distribution for a narrow resonance or bound state (taking in this last case $\Gamma_{X} \rightarrow 0$ ). We then define this combination as the spectral function involved in the energy-dependent event distributions

$\frac{\mathrm{d} \hat{M}}{\mathrm{~d} E}=\frac{\Gamma_{X}|d(E)|^{2}}{2 \pi|\alpha|^{2}}$,

with the same expression replacing $d(E)$ by $f(E)$ if the latter function is used [3]. The normalization integral is defined as

$\mathcal{N}=\int_{-\infty}^{+\infty} \mathrm{d} E \frac{\mathrm{d} \hat{M}}{\mathrm{~d} E}$

which is equal to one for the cases mentioned before. However, this is not the case when $E_{P}$ corresponds to a virtual state or other situations for which the final-state interaction function $d(E)$ has a shape that strongly departs from a nonrelativistic Breit Wigner (which also includes a Dirac delta function in the limit $\Gamma_{X} \rightarrow 0$ ). When using $f(E)$ the integration in Eq. (52) does not converge. Then we take as integration interval [ $\left.2 E_{X}, 0\right]$ as in Ref. [3], which embraces the signal region and it is enough for a semiquantitative understanding/picture based on the near value of $\mathcal{N}$ to 1 in the bound-state case.

We consider data on event distributions for $J / \psi \pi^{+} \pi^{-}$ and $D^{0} \bar{D}^{* 0}$ from $B \rightarrow K X(3872)$ decays [14-17] and inclusive $p \bar{p}$ collisions [18]. In the $B$-decay cases the number of $B \bar{B}$ pairs produced at $\Upsilon(4 S)$ is given and we denote it by $N_{B \bar{B}}$, with the same amount of neutral and charged $B \bar{B}$ pairs produced. It is also the case that the experimental papers [14-17] include the charge-conjugated decay mode to the one explicitly indicated, a convention followed by us too.

We perform fits to the data on the $J / \psi \pi^{+} \pi^{-}$event distributions from charged $B^{+} \rightarrow K^{+} J / \psi \pi^{+} \pi^{-}$decays measured by the Belle [16] and BaBar Collaborations [14]. The predicted event number $N_{i}$ at the $i$ th bin, with the center energy $E_{i}$ and bin width $\Delta$, is given by the convolution of the decay rate in Eq. (16) times $N_{B \bar{B}} / \Gamma_{B^{+}}$with the experimental energy-resolution function $R\left(E^{\prime}, E\right)$, and integrating over the bin width. We divide Eq. (16) by $\Gamma_{B^{+}}$because all the charged $B^{+} B^{-}$pairs produced, $N_{B \bar{B}} / 2$, have decayed (an integration over time of the rate of decay is implicit. The latter is given by the product of the total width times the number of $B$ mesons decaying at a given time). In addition, one has to multiply the signal function by the experimental efficiency $\varepsilon_{J}^{(+)}$. The resulting formula is

$$
\begin{aligned}
N_{i}= & \varepsilon_{J}^{(+)} N_{B \bar{B}} \mathcal{B}_{J} \int_{E_{i}-\Delta / 2}^{E_{i}+\Delta / 2} \mathrm{~d} E^{\prime} \int_{-\infty}^{\infty} \mathrm{d} E R\left(E^{\prime}, E\right) \frac{\mathrm{d} \hat{M}}{\mathrm{~d} E} \\
& +N_{B \bar{B}} \operatorname{cog}_{\mathrm{J}} \Delta .
\end{aligned}
$$

The constant $\mathcal{B}_{J}$ attached to the signal contribution in Eq. (53) can be interpreted as the product of the double branching ratios $\operatorname{Br}\left(B^{+} \rightarrow K^{+} X\right) \operatorname{Br}\left(X \rightarrow J / \psi \pi^{+} \pi^{-}\right)$when $\mathcal{N} \cong 1$, cf. Eq. (52). In this case the product $\varepsilon_{J}^{(+)} N_{B \bar{B}} \mathcal{B}_{J}$ is directly the yield $Y_{J}$. If this is not the case this interpretation is not possible but we still call this product in the same way, though its meaning is just that of a normalization constant. In this way, we re-express Eq. (53) as

$N_{i}=Y_{J} \int_{E_{i}-\Delta / 2}^{E_{i}+\Delta / 2} \mathrm{~d} E^{\prime} \int_{-\infty}^{\infty} \mathrm{d} E R\left(E^{\prime}, E\right) \frac{\mathrm{d} \hat{M}}{\mathrm{~d} E}+N_{B \bar{B}} \operatorname{cbg}_{\mathrm{J}} \Delta$.

On the other hand, the background contribution is specified by the constant $N_{B \bar{B}} \operatorname{cbg}_{\mathrm{J}} \Delta$, which can be determined by simple eye inspection from the sidebands events around the $X$ (3872) signal region. The energy-resolution function is the Gaussian function

$R\left(E^{\prime}, E\right)=\frac{1}{\sqrt{2 \pi} \sigma} \exp \left(-\frac{\left(E^{\prime}-E\right)^{2}}{2 \sigma^{2}}\right)$.

Following Ref. [4], as also used in Ref. [3], we take $\sigma=3$ $\mathrm{MeV}$ for both BaBar [14] and Belle [16] experiments on $J / \psi \pi^{+} \pi^{-}$event distributions. We take both $\mathcal{B}_{J}$ and $\operatorname{cbg}_{J}$ to be the same in the fits to BaBar and Belle data because once we take into account the different $N_{B \bar{B}}$ for both experiments $\left(N_{B \bar{B}}=4.55 \times 10^{8}\right.$ for BaBar [14], and $N_{B \bar{B}}=6.57 \times$ $10^{8}$ for Belle [16]; see also Table 1) the yields given in the experimental papers $[14,16]$ coincide. This means that the ratio of the parameters $Y_{J}$ and $\mathrm{cbg}_{\mathrm{J}}$ for $\mathrm{BaBar}$ and Belle is the same as the quotient of their respective $N_{B \bar{B}}$. Then, after fitting the data, we will give only the values of the resulting parameters for the former.

We also consider the CDF $J / \psi \pi^{+} \pi^{-}$event distribution from inclusive $p \bar{p}$ scattering [18]. We use Eq. (22) times the integrated luminosity $\mathcal{L}$, which for Ref. [18] is $2.4 \mathrm{fb}^{-1}$. In addition we neglect the $Q^{2}$ dependence except for $d(E)$ and after including the bin width, experimental efficiency $\varepsilon_{J}^{(p)}$, energy resolution and background we have

$$
\begin{aligned}
N_{i}= & \varepsilon_{J}^{(p)} \mathcal{L} \sigma_{p \bar{p} \rightarrow X} \operatorname{All} \operatorname{Br}\left(X \rightarrow J / \psi \pi^{+} \pi^{-}\right) \\
& \times \int_{E_{i}-\Delta / 2}^{E_{i}+\Delta / 2} \mathrm{~d} E^{\prime} \int_{-\infty}^{\infty} \mathrm{d} E R_{p \bar{p}}\left(E^{\prime}, E\right) \frac{\mathrm{d} \hat{M}(E)}{\mathrm{d} E} \\
& +\zeta+\varrho E_{i} .
\end{aligned}
$$

Here the bin width $\Delta$ is $1.25 \mathrm{MeV}$ and the background in the $X(3872)$ region has been parameterized as a straight line (which is easily determined from the sideband events), following the outcome in Fig. 1 of the CDF Collaboration paper 
Table 1 The $B$ decays into $K J / \psi \pi^{+} \pi^{-}$and $D^{0} \bar{D}^{* 0}$ channels are both measured by the BaBar $[14,15]$ and Belle Collaborations $[16,17]$. The total number of $B \bar{B}$ pairs $\left(N_{B \bar{B}}\right)$, bin width $(\Delta)$, and the Gaussian width $(\sigma)$ used in the experimental resolution function are given. The number of points included in the fits are also indicated. For the inclusive $p \bar{p}$ collision measured by the CDF Collaboration [18] we account for similar parameters, but now the luminosity $(\mathcal{L})$ is given instead of $N_{B \bar{B}}$. For more details see the text

\begin{tabular}{lll}
\hline$B \rightarrow K X$ & BaBar $[14,15]$ & Belle [16,17] \\
\hline$D^{0} \bar{D}^{* 0}$ & $N_{B \bar{B}}=3.83 \times 10^{8}, \Delta=2 \mathrm{MeV}$ & $N_{B \bar{B}}=6.57 \times 10^{8}, \Delta=2 \mathrm{MeV}$ \\
& $\sigma\left(E_{\exp }\right)=\sqrt{\left(0.031 \mathrm{MeV} E_{\exp }\right)}, 50$ points & $\sigma\left(E_{\exp }\right)=\sqrt{\left(0.031 \mathrm{MeV} E_{\text {exp }}\right)}, 50$ points \\
$J / \psi \pi^{+} \pi^{-}$ & $N_{B \bar{B}}=4.55 \times 10^{8}, \Delta=5 \mathrm{MeV}$ & $N_{B \bar{B}}=6.57 \times 10^{8}, \Delta=2.5 \mathrm{MeV}$ \\
& $\sigma=3 \mathrm{MeV}, 20$ points & $\sigma=3 \mathrm{MeV}, 40$ points \\
$p \bar{p}$ & $\mathrm{CDF}[18]$ & \\
& $\mathcal{L}=2.4 \mathrm{fb}^{-1}, \Delta=1.25 \mathrm{MeV}$ & \\
& $\sigma_{1}=3.2 \mathrm{MeV}, \sigma_{2}=2 \sigma_{1}, 37$ points & \\
\hline
\end{tabular}

[18]. In this reference the experimental resolution function is expressed as the sum of two Gaussians

$$
\begin{aligned}
R_{p \bar{p}}\left(E^{\prime}, E\right)= & \frac{2 / 3}{\sqrt{2 \pi} \sigma_{1}} \exp \left(-\frac{\left(E^{\prime}-E\right)^{2}}{2 \sigma_{1}^{2}}\right) \\
& +\frac{1 / 3}{\sqrt{2 \pi} \sigma_{2}} \exp \left(-\frac{\left(E^{\prime}-E\right)^{2}}{2 \sigma_{2}^{2}}\right), \\
\sigma_{1}= & 3.2 \mathrm{MeV}, \sigma_{2} \simeq 2 \sigma_{1} .
\end{aligned}
$$

Again, when $\mathcal{N} \approx 1$ the product $\varepsilon_{J}^{(p)} \sigma_{p \bar{p} \rightarrow X \operatorname{All}} \operatorname{Br}(X \rightarrow$ $J / \psi \pi^{+} \pi^{-}$) can be directly interpreted as the yield $Y_{J}^{(p)}$ but, if not, we keep this notation. We then rewrite Eq. (56) as

$$
\begin{aligned}
N_{i}= & Y_{J}^{(p)} \int_{E_{i}-\Delta / 2}^{E_{i}+\Delta / 2} \mathrm{~d} E^{\prime} \int_{-\infty}^{\infty} \mathrm{d} E R_{p \bar{p}}\left(E^{\prime}, E\right) \frac{\mathrm{d} \hat{M}(E)}{\mathrm{d} E} \\
& +\zeta+\varrho E .
\end{aligned}
$$

Concerning the $D^{0} \bar{D}^{* 0}$ event distributions from charged and neutral $B \rightarrow K X$ decays, $D^{* 0}$ is fully reconstructed from its decay products $D^{0} \pi^{0}$ and $D^{0} \gamma$ in the data from BaBar [15]. In the case of Belle data [17] we employ the one in which $D^{* 0}$ is reconstructed only from its decay product $D^{0} \pi^{0}$, because it has a much smaller background than for $D^{0} \gamma$. To reproduce the event distributions we employ the decay rate of Eq. (33) and take into account the experimental resolution, efficiency, bin width and background contributions, similarly as done for Eq. (53) above. We end with the expression

$$
\begin{aligned}
N_{i}= & \int_{E_{i}-\Delta / 2}^{E_{i}+\Delta / 2} \mathrm{~d} E^{\prime} \int_{0}^{\infty} \mathrm{d} \mathcal{E}^{\prime} R\left(E^{\prime}, \mathcal{E}^{\prime}\right) \sqrt{\mathcal{E}^{\prime}} \\
& \times\left[\frac{Y_{D}}{\sqrt{2} \pi \sqrt{E_{X}+\sqrt{E_{X}^{2}+\Gamma_{*}^{2} / 4}}}\right. \\
& \left.\times \int_{-\infty}^{\infty} \mathrm{d} E \frac{\mathrm{d} \hat{M}}{\mathrm{~d} E} \frac{\Gamma_{*}}{\left|\mathcal{E}^{\prime}-E-i \Gamma_{*} / 2\right|^{2}}+N_{B \bar{B}} \operatorname{cbg}_{\mathrm{D}}\right] .
\end{aligned}
$$

In this equation the background contribution is parameterized as $\operatorname{cbg}_{\mathrm{D}} \sqrt{\mathcal{E}^{\prime}}$ as in Ref. [3], giving rise after fitting to similar background contributions as the ones in the experimental papers Refs. $[15,17]$ (though they are parameterized in somewhat different form). The constant $\mathrm{cbg}_{\mathrm{D}}$ can easily be determined from the events above the $X$ (3872) signal region which gives rise to a rather structureless pattern. The constant $Y_{D}$ can be interpreted again as a yield for $\mathcal{N} \approx 1$ because, when integrating in $E^{\prime}$ over all the energy range the signal contribution in Eq. (59), the denominator below $Y_{D}$ is canceled because of Eq. (29). We again follow Refs. [3,4], as well as the Belle experimental analysis [17], and take the Gaussian width $\sigma$ in the resolution function $R\left(E^{\prime}, \mathcal{E}^{\prime}\right)$, Eq. (55), to be energy dependent and given by the expression

$\sigma\left(\mathcal{E}^{\prime}\right)=\sqrt{(0.031 \mathrm{MeV}) \mathcal{E}^{\prime}}$

with $\mathcal{E}^{\prime}$ running through the values in Eq. (59). For the $D^{0} \bar{D}^{* 0}$ event distributions the number of $B \bar{B}$ pairs produced is $N_{B \bar{B}}=3.83 \times 10^{8}$ for BaBar [15], and $N_{B \bar{B}}=6.57 \times 10^{8}$ for Belle [17], as also indicated in Table 1. For this case we have to take different values for the yields and background constants for fitting the BaBar and Belle data.

In all our formulas for the event distributions in Eqs. (54), (58) and (59) the background contribution is added incoherently because it is mostly combinatorial. This is the same treatment as performed in the experimental papers [14-17] as well as in the phenomenological analysis [1-5]. In a Laurent expansion of the signal amplitude around the $X(3872)$ non-resonant terms appear that add coherently but they are accounted for by the function $d(E)$ in the near-threshold region, which, as discussed in Sect. 4, is assumed to have the main dynamical features. Reference [12] attempts to unveil further dynamical information from the $B \rightarrow K D^{0} \bar{D}^{* 0}$ event distributions by considering them in a broader energy region beyond the $X(3872)$ signal and explicitly including the $D^{+} D^{*-}$ channel within the formalism. Nonetheless, the present experimental uncertainty avoids extracting any def- 

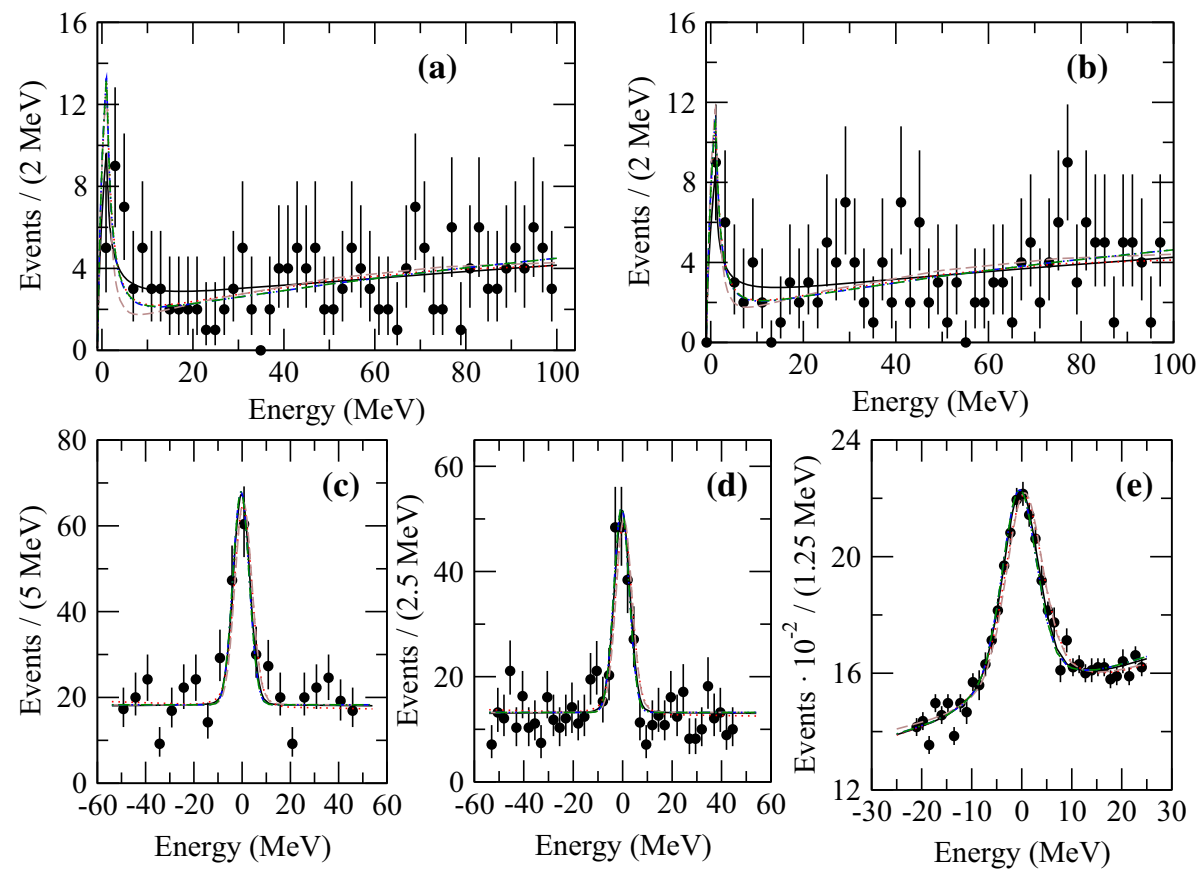

Fig. 4 Fit results for the different event distributions as a function of energy. Panels a and $\mathbf{b}$ denote the mode $D^{0} \bar{D}^{* 0}$ from BaBar [15] and Belle [17], respectively. Panels c-e denote the mode $J / \psi \pi^{+} \pi^{-}$from BaBar [14], Belle [16], and CDF [18] Collaborations, respectively. The black solid lines correspond to case 1 that employs $f(E)$. The CDD pole contribution is included in the expression of $d(E)$, and the rest of lines refer to cases making use of this parameterization. Case 2.I is shown by the red dotted lines, and case 2.II by the brown dashed lines, while cases 3.I and 3.II are given by the blue dash-double-dotted and green dash-dotted lines, in order. Part of the lines overlap so much that it is difficult to distinguish between them in the scale of the plots inite conclusion beyond the smooth background out of the $X(3872)$ region.

For the fitting process it is advantageous to rewrite Eqs. (54), (58) and (59) by using directly $|d(E)|^{2}$ instead of $\mathrm{d} \hat{M}(E) / \mathrm{d} E$, and re-absorbing the factor $1 /|\alpha|^{2}$ in the normalization constant. In this form one avoids working out the dependence of $\alpha$ on the pole position, which numerically is very convenient since it is not known a priori where the pole lies when using $d(E)$. Once the fit is done one can actually calculate $\alpha$, cf. Eq. (42), and from its value and the fitted constant the corresponding yield. This technicality is discussed in more detail in Appendix A.

\section{Combined fits}

The data sets that we include in the fits were already introduced in Sect. 5. A summary of their main characteristics can be found in Table 1. Apart from the data on $B \rightarrow K X$ decays we also include the high statistics $J / \psi \pi^{+} \pi^{-}$event distribution from $p \bar{p}$ collisions at $\sqrt{s}=1.96 \mathrm{TeV}$, measured by the CDF Collaboration [18], which also has the smallest bin width. In this way one can reach from these data a better determination of $E_{R}$. The value given by this collaboration for the $X(3872)$ mass is $M_{X}=3871.61 \pm 0.22 \mathrm{MeV}$, from which we infer $E_{R}=-0.20 \pm 0.22 \mathrm{MeV}$, that has a smaller uncertainty than the one obtained in $B$ decays from Refs. [14-17]. From the point of view of mutual compatibility between data sets [1] it is also interesting to perform a simultaneous fit to all the data on $B^{+} \rightarrow K^{+} J / \psi \pi^{+} \pi^{-}$, $B^{+}\left(B^{0}\right) \rightarrow K^{+}\left(K^{0}\right) D^{0} \bar{D}^{* 0}$ and $J / \psi \pi^{+} \pi^{-}$from inclusive $p \bar{p}$ collisions.

Experimental data points have typically asymmetric error bars; see e.g. the data points in Fig. 4. Thus, as done in Ref. [3] and also in other experimental analyses, the best values for the free parameters are determined by using the binned maximum likelihood method, which is also more appropriate in statistics than the $\chi^{2}$ method for bins with low statistics. At each bin, the number of events is assumed to obey a Poisson distribution, so that the predicted event numbers in Eqs. (54), (58) and (59) are the corresponding mean value at the bin $\left(N_{i}\right)$, while the experimentally measured number is called $Y_{i}$ (experimental data). The Poisson distribution at each bin reads $L_{i}\left(N_{i}, Y_{i}\right)=N_{i}^{Y_{i}} \exp \left(-N_{i}\right) / Y_{i}$ ! and the total probability function for a data sample is given by their products. One wants to maximize its value so that the function to be minimized is defined as

$$
-2 \log (L)=-2 \sum_{i}^{n}\left(Y_{i} \log \left(N_{i}\right)-N_{i}\right) .
$$


Fig. 5 Detail of the fit results for the near-threshold region in the $D^{0} \bar{D}^{* 0}$ event distributions. The left panel correspond to the data from the BaBar Collaboration [15] and the right one to that of the Belle Collaboration [17]. The types of lines employed to plot the boxes for every scenario are the same as in Fig. 4

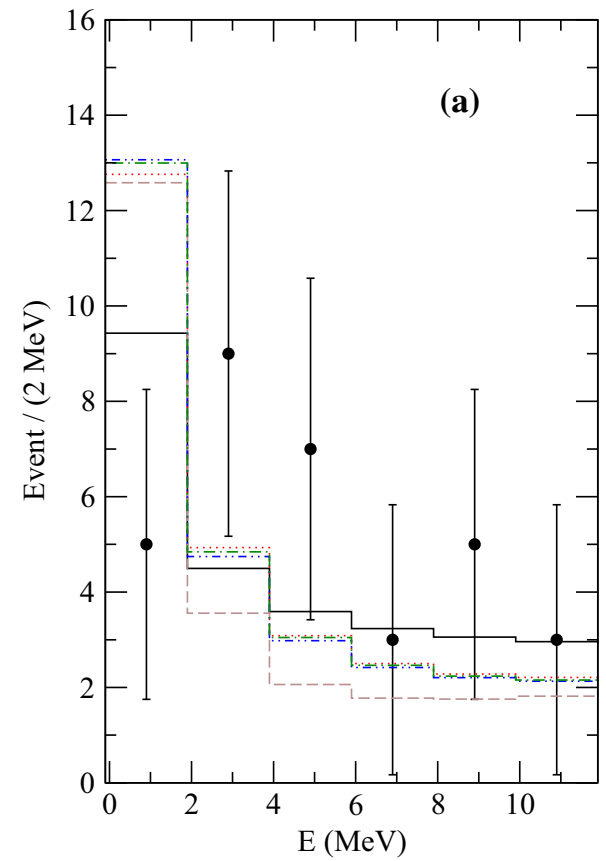

When including a CDD pole in the expression for $t(E)$, Eq. (36), one has to fix three free parameters to characterize the interaction, namely, $\lambda, M_{\mathrm{CDD}}$ and $\beta$. However, this is a too numerous set of free parameters to be fitted in terms of the data taken (additionally we have the normalization and background constants). This manifests in the fact that there are many local minima when minimizing $-2 \log L$, so that it is not clear how to extract any useful information. Instead, we have decided to consider five interesting and different possible scenarios (cases 1, 2.I-II and 3.I-II), so that each of them gives rise to an acceptable reproduction of the line shapes but corresponds to quite a different picture for $X(3872)$. In addition, we also think that the pole arrangements that result in every case are worth studying for general interest on nearthreshold states. For every of these cases studied the number of free parameters associated with $d(E)$ is one (only case 2.II below has two free parameters), so that the interaction is well constrained by data.

We gather together similar sets of information for each scenario, so that the comparison between them is more straightforward. The reproduction of the data fitted for all the cases is shown in Fig. 4 by the black solid (case 1), red dotted (case 2.I), brown dashed (case 2.II), blue dash-doubledotted (case 3.I) and green dash-dotted (case 3.II) lines. A detailed view of the more interesting near-threshold region for the $D^{0} \bar{D}^{* 0}$ event distributions is given in the histogram of Fig. 5. We also show separately the reproduction of the $J / \psi \pi^{+} \pi^{-}$event distribution of the CDF Collaboration data [18] in Fig. 9. In this figure we include the error bands too, in order to show the typical size of the uncertainty in the line shapes that stems from the systematic errors in our fits. For all the figures we follow the same convention for the meaning of the different lines. The spectroscopical information is gathered in Table 2, where we give from left to right the near-threshold pole positions, the compositeness for the bound-state pole (if present), the residues to $D^{0} \bar{D}^{* 0}$ and the yields. Finally, we show in Table 3 the scattering parameters characterizing the partial wave $t(E)$ that result from the fits. In the two rightmost columns we give the scattering length and the effective range.

All fitted parameters are given in Eqs. (62)-(64), (66) for cases 1, 2.I-II and 3.I-II, in order. The best values of the parameters are obtained by the routine MINUIT [39]. The error for a given parameter is defined as the change of that parameter that makes the function value $-2 \log L$ less than $-2 \log L_{\min }+1$ (one standard deviation), where $-2 \log L_{\min }$ is the minimum value. 
Case 1: $\quad \gamma=18.97_{-0.19}^{+0.49} \mathrm{MeV}$,

10 parameters $Y_{D 1}=7.49_{-0.41}^{+0.71}$,

$$
\begin{array}{ll}
Y_{D 1}=7.49_{-0.41}^{+0.71}, & N_{B \bar{B} ; D}^{\mathrm{BaBar}} \operatorname{cbg}_{\mathrm{D} 1}=0.18_{-0.02}^{+0.02} \mathrm{MeV}^{-3 / 2}, \\
Y_{D 2}=6.45_{-0.47}^{+0.32}, & N_{B \bar{B} ; D}^{\mathrm{BaBar}} \operatorname{cbg}_{\mathrm{D} 2}=0.11_{-0.01}^{+0.01} \mathrm{MeV}^{-3 / 2}, \\
Y_{J}=79.03_{-6.11}^{+5.65}, & N_{B \bar{B} ; J}^{\mathrm{BaBar}} \operatorname{cbg}_{\mathrm{J}}=3.59_{-0.13}^{+0.13} \mathrm{MeV}^{-1}, \\
Y_{J}^{(p)}=5.23_{-0.11}^{+0.07} \times 10^{3}, & \zeta=1515.44_{-7.40}^{+7.40} \mathrm{MeV}^{-1}, \quad \varrho=5.23_{-0.48}^{+0.48} \mathrm{MeV}^{-2} .
\end{array}
$$$$
Y_{J}=79.03_{-6.11}^{+5.65}, \quad N_{B \bar{B} ; J}^{\mathrm{BaBar}} \operatorname{cbg}_{\mathrm{J}}=3.59_{-0.13}^{+0.13} \mathrm{MeV}^{-1},
$$

Case 2.I:

$E_{R}=-0.53_{-0.11}^{+0.09} \mathrm{MeV}$

$M_{\mathrm{CDD}}=-12.29_{-1.14}^{+1.11} \mathrm{MeV}$

11 parameters: $Y_{D 1}=83.13_{-16.15}^{+22.42}$,

$N_{B \bar{B} ; D}^{\mathrm{BaBar}} \operatorname{cbg}_{\mathrm{D} 1}=0.24_{-0.02}^{+0.02} \mathrm{MeV}^{-3 / 2}$,

$Y_{D 2}=68.84_{-7.25}^{+11.86}$

$N_{B \bar{B} ; D}^{\mathrm{BaBar}} \operatorname{cbg}_{\mathrm{D} 2}=0.14_{-0.01}^{+0.01} \mathrm{MeV}^{-3 / 2}$,

$Y_{J}=8.44_{-2.59}^{+3.64} \times 10^{3}$,

$N_{B \bar{B} ; J}^{\mathrm{BaBar}} \operatorname{cbg}_{\mathrm{J}}=3.46_{-0.14}^{+0.14} \mathrm{MeV}^{-1}$,

$Y_{J}^{(p)}=5.78_{-1.64}^{+2.29} \times 10^{5}$,

$$
\zeta=1498.57_{-9.73}^{+9.73} \mathrm{MeV}^{-1}, \quad \varrho=5.09_{-0.49}^{+0.49} \mathrm{MeV}^{-2} \text {. }
$$

Case 2.II

$$
E_{R}=-0.95_{-0.05}^{+0.07} \mathrm{MeV},
$$

10 parameters

$$
\begin{array}{ll}
Y_{D 1}=79.75_{-19.81}^{+22.46}, & N_{B \bar{B} ; D}^{\mathrm{BaBar}} \operatorname{cog}_{\mathrm{D} 1}=0.25_{-0.02}^{+0.02} \mathrm{MeV}^{-3 / 2}, \\
Y_{D 2}=72.39_{-8.02}^{+9.18}, & N_{B \bar{B} ; D}^{\mathrm{BaBar}} \operatorname{cbg}_{\mathrm{D} 2}=0.15_{-0.01}^{+0.01} \mathrm{MeV}^{-3 / 2}, \\
Y_{J}=9.23_{-1.57}^{+1.60} \times 10^{3}, & N_{B \bar{B} ; J}^{\mathrm{BaBar}} \operatorname{cbg}_{\mathrm{J}}=3.65_{-0.13}^{+0.13} \mathrm{MeV}^{-1}, \\
Y_{J}^{(p)}=6.23_{-0.84}^{+0.71} \times 10^{5}, & \zeta=1520.23_{-8.57}^{+8.57} \mathrm{MeV}^{-1}, \quad \varrho=4.48_{-0.50}^{+0.50} \mathrm{MeV}^{-2} .
\end{array}
$$

Case 3.I: $\quad E_{R}=-0.49_{-0.03}^{+0.04} \mathrm{MeV}$,

10 parameters

$$
\begin{array}{ll}
Y_{D 1}=25.45_{-4.15}^{+4.05}, & N_{B \bar{B} ; D}^{\mathrm{BaBar}} \operatorname{cbg}_{\mathrm{D} 1}=0.22_{-0.02}^{+0.02} \mathrm{MeV}^{-3 / 2}, \\
Y_{D 2}=21.08_{-1.89}^{+1.32}, & N_{B \bar{B} ; D}^{\mathrm{BaBa}} \operatorname{cbg}_{\mathrm{D} 2}=0.13_{-0.01}^{+0.01} \mathrm{MeV}^{-3 / 2}, \\
Y_{J}=80.14_{-5.19}^{+5.67}, & N_{B \bar{B} ; J}^{\mathrm{BaBar}} \operatorname{cbg}_{\mathrm{J}}=3.66_{-0.13}^{+0.13} \mathrm{MeV}^{-1}, \\
Y_{J}^{(p)}=5.26_{-0.08}^{+0.12} \times 10^{3}, & \zeta=1524.70_{-7.91}^{+7.91} \mathrm{MeV}^{-1}, \quad \varrho=5.32_{-0.49}^{+0.49} \mathrm{MeV}^{-2} .
\end{array}
$$

Case 3.II: $\quad E_{R}=-0.49_{-0.01}^{+0.02} \mathrm{MeV}$,

10 parameters:

$$
\begin{array}{ll}
Y_{D 1}=22.90_{-3.02}^{+2.94}, & N_{B \bar{B} ; D}^{\mathrm{BaBar}} \operatorname{cbg}_{\mathrm{D} 1}=0.22_{-0.02}^{+0.02} \mathrm{MeV}^{-3 / 2}, \\
Y_{D 2}=18.92_{-0.77}^{+1.40}, & N_{B \bar{B} ; D}^{\mathrm{BaBa}} \operatorname{cbg}_{\mathrm{D} 2}=0.13_{-0.01}^{+0.01} \mathrm{MeV}^{-3 / 2}, \\
Y_{J}=80.07_{-5.36}^{+5.14}, & N_{B \bar{B} ; J}^{\mathrm{BaBar}} \operatorname{cbg}_{\mathrm{J}}=3.66_{-0.13}^{+0.13} \mathrm{MeV}^{-1}, \\
Y_{J}^{(p)}=5.28_{-0.17}^{+0.05} \times 10^{3}, & \zeta=1524.66_{-7.73}^{+7.73} \mathrm{MeV}^{-1}, \quad \varrho=5.32_{-0.49}^{+0.49} \mathrm{MeV}^{-2}
\end{array}
$$

\subsection{Case 1: Bound state}

In this first case we fit the different data sets by using the function $f(E)[2,3]$, in order to take into account the FSI between $\bar{D}^{0} D^{* 0}$, while Ref. [3] makes fits to different data sets separately. We also include this standard case as a reference to compare with other less standard ones introduced below. As mentioned above, the inverse scattering length $\gamma$ in the expression of $f(E)$ can be taken complex (with negative imaginary part) to mimic inelastic channels. Indeed complex values were used in Ref. [3], though it was found that the experimental data can be equally well described by taking the imaginary part of $\gamma$ free or fixing it to 0 (as we have also found). Physically, this indicates that an inelastic effect, as the transition $D^{0} \bar{D}^{* 0} \rightarrow J / \psi \pi^{+} \pi^{-}$, has little impact on FSI and we fix it always to zero in our fits, which are also checked to be stable if the imaginary part of $\gamma$ is released. 
Table 2 Summary of the combined fits in Sect. 6 for all the cases. From left to right, the pole positions, compositeness $(X)$, residue and yields $(Y)$ are given. $Y_{D 1}, Y_{D 2}$ denote the yields corresponding to the $\mathrm{BaBar}$ and Belle data on the $D^{0} \bar{D}^{* 0}$ mode, respectively; $Y_{J}$ denotes the one for BaBar on the $J / \psi \pi^{+} \pi^{-}$mode and $Y_{J}^{(p)}$ applies to the CDF Collaboration data with $p \bar{p}$ collisions. The normalization for the residues is such that $\Gamma=k g^{2} /\left(8 \pi M_{X}^{2}\right)$ as in Ref. [19]

\begin{tabular}{|c|c|c|c|c|}
\hline Cases & Pole position $[\mathrm{MeV}]$ & $X$ & Residue $\left[\mathrm{GeV}^{2}\right]$ & $\begin{array}{l}Y_{D 1} \\
Y_{D 2} \\
Y_{J} \\
Y_{J}^{(p)} \\
\end{array}$ \\
\hline 1 & $-0.19_{-0.01}^{+0.01}-i 0.0325$ & 1.0 & $14.78_{-0.14}^{+0.38}$ & $\begin{array}{l}7.49_{-0.41}^{+0.71} \\
6.45_{-0.47}^{+0.32} \\
79.03_{-6.11}^{+5.65} \\
5.23_{-0.11}^{+0.07} \times 10^{3}\end{array}$ \\
\hline 2.I & $\begin{array}{l}-0.36_{-0.10}^{+0.08}-i 0.18_{-0.02}^{+0.01} \\
-0.70_{-0.13}^{+0.11}+i 0.17_{-0.01}^{+0.02}\end{array}$ & & $\begin{array}{c}-47.48_{-12.40}^{+9.75}-i 66.06_{-13.50}^{+10.87} \\
82.69_{-11.88}^{+14.84}+i 66.03_{-10.87}^{+13.50}\end{array}$ & $\begin{array}{l}83.13_{-16.15}^{+22.42} \\
40.13_{-7.25}^{+11.86} \\
8.44_{-2.59}^{+3.64} \times 10^{3} \\
5.78_{-1.65}^{+2.29} \times 10^{5}\end{array}$ \\
\hline 2.II & $\begin{array}{l}-0.33_{-0.03}^{+0.04}-i 0.31_{-0.01}^{+0.02} \\
-0.84_{-0.05}^{+0.07}+i 0.77_{-0.04}^{+0.03} \\
-1.67_{-0.08}^{+0.10}-i 0.49_{-0.02}^{+0.02}\end{array}$ & & $\begin{array}{l}-6.24_{-2.20}^{+2.80}-i 1.41_{-0.10}^{+0.14} \times 10^{2} \\
\left(2.32_{-0.21}^{+0.16}-i 1.77_{-0.08}^{+0.11}\right) \times 10^{2} \\
\left(-3.26_{-0.16}^{+0.22}+i 3.18_{-0.25}^{+0.18}\right) \times 10^{2}\end{array}$ & $\begin{array}{l}79.75_{-19.81}^{+22.46} \\
42.20_{-8.02}^{+9.18} \\
9.23_{-1.57}^{+1.60} \times 10^{3} \\
6.23_{-0.84}^{+0.71} \times 10^{5}\end{array}$ \\
\hline 3.I & $\begin{array}{l}-0.50_{-0.03}^{+0.04} \\
-0.68_{-0.03}^{+0.05}\end{array}$ & $0.061_{-0.002}^{+0.003}$ & $\begin{array}{r}1.52_{-0.01}^{+0.01} \\
2.72_{-0.04}^{+0.02}\end{array}$ & $\begin{array}{l}25.45_{-4.15}^{+4.05} \\
12.29_{-1.89}^{+1.32} \\
80.14_{-5.19}^{+5.67} \\
5.26_{-0.08}^{+0.12} \times 10^{3}\end{array}$ \\
\hline 3.II & $\begin{array}{l}-0.51_{-0.01}^{+0.03} \\
-1.06_{-0.02}^{+0.05}\end{array}$ & $0.158_{-0.001}^{+0.001}$ & $\begin{array}{l}3.96_{-0.08}^{+0.03} \\
7.56_{-0.20}^{+0.08}\end{array}$ & $\begin{array}{l}22.90_{-3.02}^{+2.94} \\
11.03_{-0.77}^{+1.40} \\
80.07_{-5.36}^{+5.14} \\
5.28_{-0.17}^{+0.05} \times 10^{3}\end{array}$ \\
\hline
\end{tabular}

The parameters corresponding to the yields and background constants are $Y_{J}, Y_{J}^{(p)}, Y_{D}, \operatorname{cbg}_{\mathrm{J}}, \xi, \rho$ and $\operatorname{cbg}_{\mathrm{D}}$. The most interesting free parameters are those fixing the interaction $t(E)$, which for the present case just reduces to the inverse scattering length $\gamma$. The background constants can be determined rather straightforwardly, because the background is very smooth in all cases and fixed by the sidebands events around the signal region. The best fitted parameters that we obtain for case 1 are given in Eq. (62). The reproduction of the event distributions is shown in Figs. 4 and 5 by the black solid lines. The different yields, which we denote globally as $Y_{F}$ in the following, can be interpreted properly in this way because $\mathcal{N}=0.98 \simeq 1$, as expected for a bound state.

With the values of the parameters at hand in Eq. (62), $X(3872)$ is a near-threshold bound-state pole in the function $f(E)$ located at $-0.19_{-0.01}^{+0.01}-i \Gamma_{*} / 2 \mathrm{MeV}$. Here the imaginary part stems purely from the finite width of the constituent $D^{* 0}$. As a result the scattering length is large and positive with the value $a=10.40_{-0.26}^{+0.10} \mathrm{fm}$.

$X$, of the resulting bound-state pole [40-42] can be written as [8]
$X=-i g_{k}^{2}$,

with $g_{k}^{2}$ the residue of the amplitude in the momentum variable $k$. For $f(E)=1 /(-1 / a-i k)$ its residue at the pole position in the variable $k$ is $i$, so that $X=1$. That is, independently of which is the dynamical seed for binding (origin of $\gamma$ ) this is a bound state whose composition is exhausted by the $D^{0} \bar{D}^{* 0}$ component $[2,3,11]$. This result is in agreement with Ref. [6], which concludes that the scattering length approximation is only valid for the bound-state case if its compositeness is 1 . We also give in the fourth column of Table 2 the residue $g^{2}$ of the $S$-wave scattering amplitude for each near-threshold pole in a more standard normalization, in which the partial decay width of a narrow resonance is $\Gamma=k g^{2} /\left(8 \pi M_{X}^{2}\right)$ [19]. This residue for $f(E)$ reads $g^{2}=-i 16 \pi k_{P} P_{X}^{2} / \mu$.

\subsection{Case 2: Virtual state}

In the previous section, as well as in Ref. [3], only the scattering length is taken into account. However, considering the 
Table 3 Parameters

characterizing the $S$-wave interaction $t(E)$ for cases 1-3.II of the combined fits in Sect. 6 .

The ellipses indicate that it is not appropriate to give the corresponding magnitude in such case. The elementariness $Z$ is calculated from the knowledge of the bound-state mass and the Weinberg's compositeness relations of Eq. (84) either in terms of $a\left(Z_{a}\right)$ or $r\left(Z_{r}\right)$. The error is not given when its estimation is smaller than the precision shown

\begin{tabular}{|c|c|c|c|c|c|}
\hline Cases & $\begin{array}{l}\lambda \\
{\left[\mathrm{MeV}^{2}\right]}\end{array}$ & $\begin{array}{l}M_{\mathrm{CDD}} \\
{[\mathrm{MeV}]}\end{array}$ & $\begin{array}{l}\beta \\
{[\mathrm{MeV}]}\end{array}$ & $\begin{array}{l}a(\mathrm{fm}) \\
Z_{a}\end{array}$ & $\begin{array}{l}r(\mathrm{fm}) \\
Z_{r}\end{array}$ \\
\hline 1 & $\ldots$ & $\ldots$ & $\ldots$ & $\begin{array}{l}10.40_{-0.26}^{+0.10} \\
0\end{array}$ & $\begin{array}{l}0 . \\
0 .\end{array}$ \\
\hline 2.I & $4176.35_{-462.56}^{+385.00}$ & $-12.29_{-1.14}^{+1.11}$ & $-323.12_{-6.01}^{+11.12}$ & $-11.82_{-1.21}^{+1.15}$ & $-5.64_{-0.61}^{+0.58}$ \\
\hline 2.II & $324.68_{-34.45}^{+26.32}$ & $2.84_{-0.20}^{+0.15}$ & $128.43_{-4.71}^{+3.38}$ & $\begin{array}{l}\cdots \\
-13.83_{-0.53}^{+0.35}\end{array}$ & $\begin{array}{l}\cdots \\
-8.19_{-0.31}^{+0.21}\end{array}$ \\
\hline 3.I & $260.46_{-2.31}^{+1.39}$ & $0.25_{-0.03}^{+0.04}$ & $317.25_{-0.40}^{+0.31}$ & $\begin{array}{l}\cdots \\
0.27_{-0.04}^{+0.07}\end{array}$ & $\begin{array}{l}\cdots \\
-847.84_{-223.69}^{+212.48}\end{array}$ \\
\hline 3.II & $2204.29_{-76.19}^{+31.04}$ & $3.21_{-0.08}^{+0.03}$ & $561.70_{-3.41}^{+1.37}$ & $\begin{array}{l}\cdots \\
1.57_{-0.02}^{+0.05} \\
0.86\end{array}$ & $\begin{array}{l}\cdots \\
-43.75_{-0.61}^{+0.24} \\
0.87\end{array}$ \\
\hline
\end{tabular}

$\lambda=\frac{1}{2 G_{R}}\left(G_{R}^{2}+4 \widetilde{\Delta}^{2}\right) v \cos u$,

$\beta=\frac{v}{G_{R}}\left(-2 \widetilde{\Delta} \cos u+G_{R} \sin u\right)$,

$u \equiv \frac{1}{2} \arg \left(E_{R}+i \frac{G_{R}}{2}\right), \quad v \equiv \sqrt{2 \mu\left(E_{R}^{2}+G_{R}^{2} / 4\right)}$,

$\widetilde{\Delta} \equiv E_{R}-M_{\mathrm{CDD}}$.

Thus, the function $d(E)$, Eq. (41), depends on $E_{R}$ and $M_{\mathrm{CDD}}$, with a stable limit for $G_{R} \rightarrow 0^{+}$. The latter can be performed algebraically from Eq. (69) with the result

$\lambda=\frac{\mu}{\varkappa}\left(M_{\mathrm{CDD}}-E_{R}\right)^{2}$,
$\beta=\frac{\mu}{\varkappa}\left(M_{\mathrm{CDD}}-3 E_{R}\right)$,

with $\varkappa=\sqrt{2 \mu\left|E_{R}\right|}$. Notice that keeping $G_{R}$ finite and later taking the limit $G_{R} \rightarrow 0^{+}$allows us to dispose of one more constraint (one free parameter less) than if we had taken directly $G_{R}=0$ and then imposed the requirement that $t_{\text {II }}\left(E_{R}\right)=0$.

Next, let us consider the secular equation for the poles of $t(E)$, Eq. (36), in the complex $k$ plane:

$\lambda+\left(E-M_{\mathrm{CDD}}\right)(-i k+\beta)=0$.

We substitute the expressions for $\lambda$ and $\beta$ of Eq. (70) in the previous equation and obtain

$(k+i \varkappa)^{2}\left(k+i \frac{\mu}{\varkappa}\left(M_{\mathrm{CDD}}+E_{R}\right)\right)=0$,

where the global factor $-i / 2 \mu$ has been dropped. This equation explicitly shows that $k=-i \varkappa$ is a double virtual-state pole.

It is also trivial from Eq. (72) to impose a triplet virtualstate pole by choosing appropriately $M_{\mathrm{CDD}}$ to

$M_{\mathrm{CDD}}=-3 E_{R}$.

In the following we denote by case 2.I the one with the double virtual-state pole and by case 2.II that with the triplet pole. 
To fit data we reinsert the finite width for $D^{* 0}$ and use the expressions for $\lambda$ and $\beta$ in Eq. (70). For case 2.I one has two free parameters $\left(E_{R}\right.$ and $\left.M_{\mathrm{CDD}}\right)$ to characterize the interaction, while for case 2.II only one free parameter remains $\left(E_{R}\right)$ because of the extra Eq. (73). The fitted parameters in each case are given in Eqs. (63) and (64).

The reproduction of the data for cases 2.I and 2.II are shown by the red dotted and brown dashed lines in Figs. 4 and 5 , respectively. There are visible differences between case 1 and cases 2.I-II, in the peak region of the $D^{0} \bar{D}^{* 0}$ and $J / \psi \pi^{+} \pi^{-}$event distributions. For the former, the scenarios 2.I-II produce a signal higher in the peak that decreases faster with energy, while for the latter there is a displacement of the peak towards the threshold in the virtual-state cases. This is more visible in Fig. 9 where we show only the reproduction of the CDF data [18] including error bands as well. The reason for this displacement is because the virtual-state poles only manifests in the physical axis above threshold, so that the peak of the event distribution happens almost on top of it. Nonetheless, we have to say that the shift in the signal peak for cases 2.I-II and the $J / \psi \pi^{+} \pi^{-}$data diminish considerably if we exclude in the fit the $D^{0} \bar{D}^{* 0}$ data from the BaBar Collaboration [15]. Furthermore, it is clear that these data give rise to a line shape with a displaced peak towards higher energies as compared with the analogous data from the Belle Collaboration [17]; see Fig. 5. Thus, it is not fair just to conclude that cases 2.I-II are not favorable because of the shift of the signal shape in the $J / \psi \pi^{+} \pi^{-}$CDF data [18] until one also disposes of better data for the $D^{0} \bar{D}^{* 0}$ event distributions.

It is clear that when taking $\Gamma_{*}=0$ (so that standard ERE is perfectly fine mathematically for $\left|k^{2}\right|<2 \mu\left|M_{\mathrm{CDD}}\right|$ ), all the near-threshold poles are at $E_{R}$. For case 2.I the CDD pole lies relatively far away from the $D^{0} \bar{D}^{* 0}$ threshold. However, if we kept only $a=-11.82 \mathrm{fm}$ in the ERE the pole position in the second RS would be $-0.14 \mathrm{MeV}$, if including $r=-5.64 \mathrm{fm}$ it is $-0.93 \mathrm{fm}$, with $v_{2}$ then it still moves to $-0.58 \mathrm{MeV}$ and with $v_{3}$ one has $-0.54 \mathrm{MeV}$. Thus, though the CDD pole is around $-12 \mathrm{MeV}$ one needs several terms in the ERE to reproduce adequately the $S$-wave amplitude. In particular, it is not enough just to keep e.g. the scattering length contribution as in case 1 or as in Ref. [2,3]. For case 2 .II the CDD pole is much closer, around $3 \mathrm{MeV}$, so that the convergence of the ERE is much worse and many more terms in the ERE should be kept to properly reproduce the pole position.

At this point it is interesting to display the pole trajectories as a function of $g_{f}$, while keeping constants $\gamma_{V}$ and $E_{f}$, cf. Eq. (47). In this way, we have a quite intuitive decoupling limit $g_{f} \rightarrow 0$ in which two poles at $\pm \sqrt{2 \mu E_{R}}$ correspond to the bare state and an additional one at $i \gamma_{V}=-i \beta$ stems from the direct coupling between the $D^{0} \bar{D}^{* 0}$ mesons. As $g_{f}$ increases an interesting interplay between the pole movements arises reflecting the coupling between the bare state and the continuum channel. For the fit of case 2.I one has the central values $g_{f 0}=0.080, \gamma_{V}=323 \mathrm{MeV}$ and $E_{f}=0.63 \rightarrow \pm \sqrt{2 \mu E_{f}}= \pm 35 \mathrm{MeV}$. Its pole trajectories, shown in the two top panels of Fig. 6, are obtained by increasing $g_{f}$ from one tenth of the fitted value up to 10 times it. In the left panel we show the global picture, including the far away bound state, while in the right panel we show a finer detail of the two near-threshold poles that stem from the bare state, which for $g_{f}=g_{f 0}$ become degenerate. For case 2.II we have the central values $g_{f 0}=0.039, \gamma_{V}=-128.6 \mathrm{MeV}$ and $E_{f}=0.32 \rightarrow \pm \sqrt{2 \mu E_{f}}= \pm 24.7 \mathrm{MeV}$. The three virtual-state poles, 2 from the bare state and another from the direct interactions between the $D^{0} \bar{D}^{* 0}$ mesons, become degenerate for $g_{f}=g_{f 0}$ and the triplet pole arises. Compared with the pole trajectories explicitly shown in Ref. [11] ours correspond to a much larger absolute value of $\gamma_{V}$ than those in Ref. [11] with $\left|\gamma_{V}\right|$ between 20-55 MeV. There is no pole trajectory with three poles merging neither in Ref. [11] nor in Ref. [45].

Due to the relationship between a near-threshold CDD pole and a bare state weakly coupled to the continuum (as exemplified explicitly in the third expression of Eq. (47)) one expects that for case 2.I the virtual-state pole has mostly a dynamical origin while for case 2.II, with a much smaller $\left|M_{\mathrm{CDD}}\right|$, one anticipates an important bare component. This expectation can be put in a more quantitative basis by using the spectral density function $\omega(E)$ as introduced in Ref. [7], which reflects the amount of the continuum spectrum in the bare state. For the dynamical model of Ref. [11] the spectral function can be calculated and reads

$$
\begin{aligned}
\omega(E) & =\theta(E) \frac{\gamma_{V}\left(E_{f}-M_{\mathrm{CDD}}\right) k / \pi}{\left|\gamma_{V}\left(E-E_{f}\right)+i\left(E-M_{\mathrm{CDD}}\right) k\right|^{2}} \\
& =\theta(E) \frac{\lambda k / \pi}{\left|\lambda+(\beta-i k)\left(E-M_{\mathrm{CDD}}\right)\right|^{2}},
\end{aligned}
$$

with $\theta(x)$ the Heaviside function. We have used the prescription argued in Ref. [7], so that the spectral density function is integrated only along the $X(3872)$ signal region, taken as $1 \mathrm{MeV}$ above threshold,

$W=\int_{0}^{1} \mathrm{~d} E \omega(E)$.

This is a reasonable interval as explicitly shown in Fig. 7, where several spectral density functions are shown for increasing $g_{f}$, from $0.1 g_{f 0}$ up to $2 g_{f 0}$, with $\gamma_{V}$ and $E_{F}$ fixed. The left panel is for case 2.I and the right one for case 2.II. In the decoupling limit the spectral density is strongly peaked and becomes more diluted as $g_{f}$ increases. The value of the integral $W$ is interpreted as the bare component in the resonance composition, and we obtain the values of $W=0.38$ for case 2.I and $W=0.75$ for case 2.II. This result is in line with our previous conclusion based on the value of $M_{\mathrm{CDD}}$, 

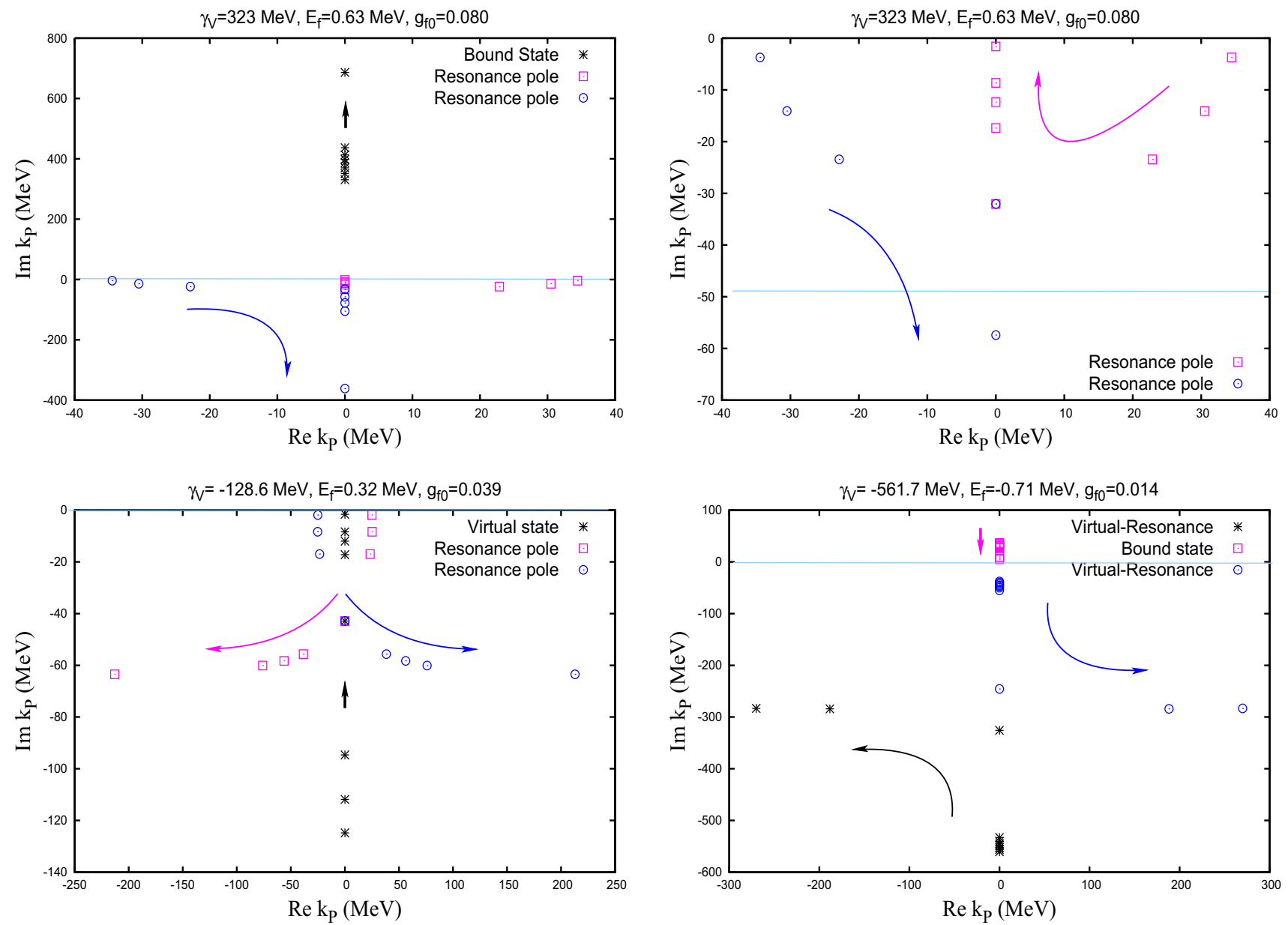

Fig. 6 Evolution of the poles as $g_{f}$ increases, with $E_{f}$ and $\gamma_{V}$ fixed. The top panels are for case 2.I, the bottom-left panel for case 2.II and the bottom-right one for case 3.II. In the right top panel a detail of the near-threshold region is given for case 2.I

since for the former case the $D^{0} \bar{D}^{* 0}$ component is dominant (around a 60\%) while for the latter is much smaller (around a $25 \%$ ). For different $g_{f}$ we also give in the legends of the panels of Fig. 7 the resulting value of $W$, that increases as $g_{f}$ decreases because the bare component is larger then. In the limit $g_{f} \rightarrow 1 \mathrm{~W}$ tends to 1 , as it should.

We now discuss until the end of this section the situation used to actually fit data with $\Gamma_{*} \neq 0$. As already discussed above for case 2.I we find two near-threshold virtual-state poles in the second RS and one deep bound state in the first $\mathrm{RS}$. The latter is driven by the large negative value of $\beta$, so that $k \approx-i \beta \cdot{ }^{10}$ For the triplet case all poles lie close to the threshold. Let us recall that the pole positions are given in the second column of Table 2. Contrary to case 1 their imaginary parts do not coincide with $\Gamma_{*} / 2$ because of the energy dependence of the CDD pole entering in $d(E)$. One can observe that the imaginary parts of the pole positions for case 2.I are much larger in absolute value than $\Gamma_{*} / 2$, and

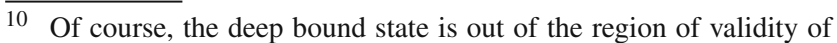
our approach and it is just referred for illustrative purposes. that for case 2.II they are even larger than for case 2.I. This noticeable fact is due to the dependence on

$\rho=\frac{\Gamma_{*}}{E_{R}}$,

which shows a striking non-analytic behavior because of the higher order of the virtual-state pole in the limit $\Gamma_{*} \rightarrow 0$, and corrections to the pole positions are controlled by $\rho^{1 / n}$ with $n=2$ and 3 for the double and triplet virtual-state poles, respectively. This implies that these corrections are significantly larger than expected as the order of the pole increases. Of course, this is exemplified by the given splitting in the pole positions for the double and triplet poles (being correspondingly larger for the latter). The dependence of the pole positions with $\rho$ is worked out explicitly in Appendix B and we give here the final results.

For case 2.I we can simplify formulas by taking into account that $\left|M_{\mathrm{CDD}}\right| \gg\left|E_{R}\right|$. The poles are located at

$$
k_{1,2}=-i \varkappa\left(1 \pm \frac{1-i}{2} \rho^{\frac{1}{2}}\right) \text {, }
$$$$
k_{3}=-i \beta,
$$ 

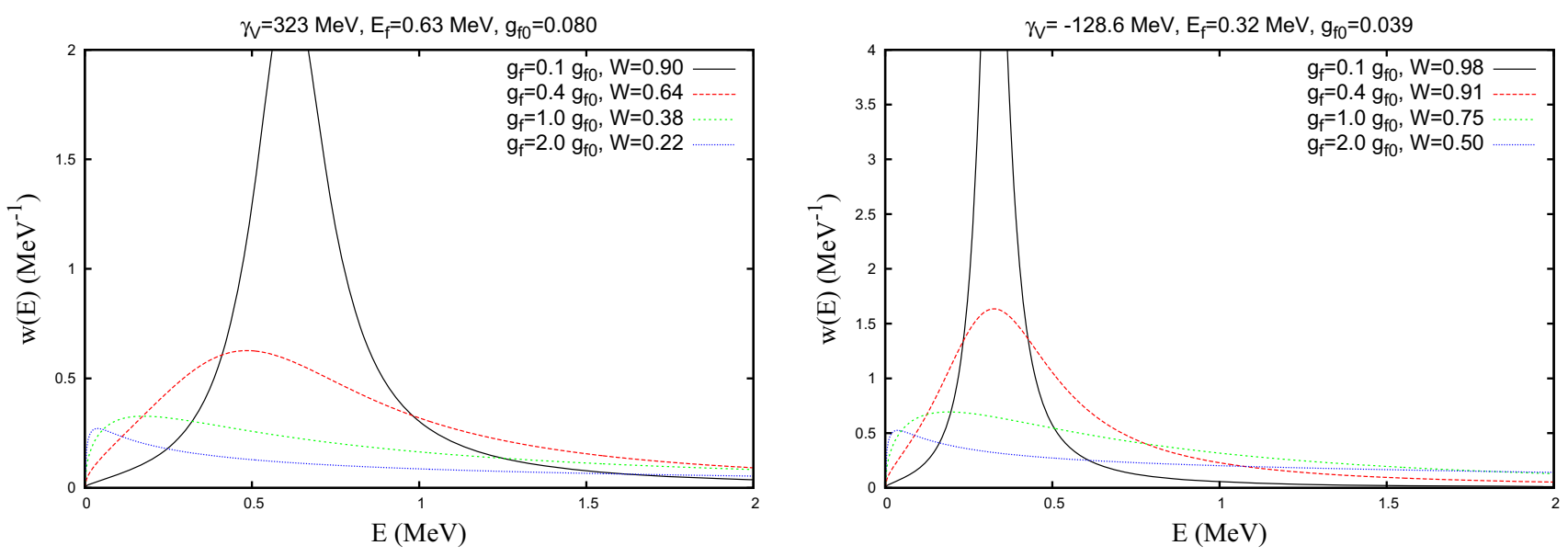

Fig. 7 Spectral density function for case 2.I (left) and case 2.II (right panel) for several values of $g_{f}$, with $E_{f}$ and $\gamma_{V}$ fixed. The value of $W$ is also indicated in the legends for each entry of $g_{f}$

with $\varkappa=\sqrt{2 \mu\left|E_{R}\right|}$. Their positions in the energy plane, $E=k^{2} / 2 \mu-i \Gamma_{*} / 2$, are

$$
\begin{aligned}
E_{1,2} & =E_{R}\left(1 \pm \rho^{\frac{1}{2}}(1-i)\right), \\
E_{3} & =-\frac{\beta^{2}}{2 \mu}-i \frac{\Gamma_{*}}{2} .
\end{aligned}
$$

For the triplet virtual-state pole in case 2.II, we have the pole positions

$$
\begin{aligned}
& k_{1}=-i \varkappa-\varkappa \rho^{\frac{1}{3}}, \\
& k_{2}=-i \varkappa\left(1+\frac{\sqrt{3}}{2} \rho^{\frac{1}{3}}\right)+\frac{\varkappa}{2} \rho^{\frac{1}{3}}, \\
& k_{3}=-i \varkappa\left(1-\frac{\sqrt{3}}{2} \rho^{\frac{1}{3}}\right)+\frac{\varkappa}{2} \rho^{\frac{1}{3}},
\end{aligned}
$$

which imply the energies

$$
\begin{aligned}
& E_{1}=E_{R}\left(1-i 2 \rho^{\frac{1}{3}}\right), \\
& E_{2}=E_{R}\left(1+\frac{\sqrt{3}}{2} \rho^{\frac{1}{3}}\right)\left(1+\frac{\sqrt{3}}{2} \rho^{\frac{1}{3}}+i \rho^{\frac{1}{3}}\right), \\
& E_{3}=E_{R}\left(1-\frac{\sqrt{3}}{2} \rho^{\frac{1}{3}}\right)\left(1-\frac{\sqrt{3}}{2} \rho^{\frac{1}{3}}+i \rho^{\frac{1}{3}}\right) .
\end{aligned}
$$

Higher orders in $\rho^{\frac{1}{3}}$ have been neglected in Eqs. (79) and (80)

As noticed above, because of this non-analytic behavior in $\rho$, the imaginary parts for the pole positions in energy, except for $E_{3}$ in case 2.I which is just a simple pole in the limit $\Gamma_{*}=0$, are much larger in absolute value than a naive estimation from the width of the constituent $D^{* 0}$. In particular, one can immediately deduce from Eq. (78) that the imaginary parts have opposite signs for the poles $E_{1,2}$ of case 2.I. For case 2.II it follows from Eq. (80) that the pole at $E_{1}$ has a positive imaginary part while the latter is negative for both poles at $E_{2}$ and $E_{3}$. As far as we know this is the first time that it is noticed such non-analytic behavior of the pole positions in the width of one of its constituents for higher degree poles. Of course, this might have important phenomenological implications. In particular, for our present analysis it favors extension of the virtual-state signal to energies above the $D^{0} \bar{D}^{* 0}$ threshold, because it increases the overlapping with the $D^{* 0}$ Lorentzian in Eq. (59). Within other context, non-analyticities of the pole positions as a function of a strength parameter near a two-body threshold around the point where the two conjugated poles meet have been derived in Refs. [45,46]. Similar behavior has also been found as a function of quark masses for chiral extrapolations [47-50].

The residues of the poles, given in the fourth column of Table 2, are very large for case 2.I and huge for case 2.II. The point is that they are affected by the extra singularity coming from the other coalescing poles in the limit $\Gamma_{*} \rightarrow 0^{+}$. For the virtual-state cases one cannot interpret the normalization constants $Y_{F}$ as yields because the virtual-state pole is below threshold in the second RS and then it is blocked by the threshold branch-point singularity, so that it does not directly influence the physical axis for $E<0$. This also manifests in that the normalization integral $\mathcal{N}$, Eq. (52), is very different from 1.

\subsection{Cases 3: Simultaneous virtual and bound state}

In this case we again use the more general parameterization based on $d(E)$ and move towards a scenario in which one finds simultaneously a bound-state pole in the first RS and a virtual-state one in the second RS. To end with such a situation we impose the requirement that in the isospin limit there is a double virtual-state pole independently of the common masses taken for the isospin multiplets (either the 
masses of the neutral or charged isospin $D^{(*)}$ members). This is a way to enforce a weak coupling of the bare states with the continuum, have poles in different RS's and end with a bound state with small compositeness (or large elementariness). At this point we adapt, as an intermediate step to end with our elastic $D^{0} \bar{D}^{* 0} S$ wave, the main ideas developed in Ref. [31]. This reference takes into account the coupledchannel structure of $\pi^{+} \Sigma_{c}^{0}, \pi^{-} \Sigma_{c}^{++}$and $\pi^{0} \Sigma_{c}^{+}$in relation with the $\Lambda_{c}(2595)^{+}$resonance, where the symbol $\Sigma_{c}$ actually refers to the $\Sigma_{c}$ (2455) [19]. However, the resulting expression reduces to that of Eq. (36) for single coupled-scattering since we focus on the $X(3872)$ signal region around the $D^{0} \bar{D}^{* 0}$ threshold, because of the same reasons as already discussed when matching our results with those of Ref. [12] in the last part of Sect. 4. Of course, these considerations translate into a different dependence of $\lambda$ and $\beta$ on $E_{R}$ than in cases 2.I-II analyzed in Sect. 6.2.

The basic strategy is the following: (i) We take the isospin limit for $D^{0} \bar{D}^{* 0}$ and $D^{+} D^{*-}$ coupled-channel scattering, with masses equal to either those of the neutral particles for each isospin doublet $\left(D^{0}\right.$ and $D^{* 0}$ ) or to the charged ones $\left(D^{+}\right.$and $\left.D^{*+}\right)$. At this early stage the zero width limit for the $D^{* 0}$ is taken. (ii) For every isospin limit defined in (i) we impose having the same virtual-state pole position located at $E_{R}-i G_{R} / 2$, taking the limit $G_{R} \rightarrow 0^{+}$at the end, similarly as done in Sect. 6.2 to end with less free parameters in a more restricted situation. (iii) The previous point provides us with four equations that are used to fix two of the three parameters in $d(E)$, Eq. (41), namely $\beta$ and $\lambda .{ }^{11}$ The remaining third parameter, $M_{\mathrm{CDD}}$, is also fixed by imposing that $d(E)^{-1}$ vanishes in the first RS below threshold at $E_{R}$. (iv) In this way all the parameters specifying $d(E)$ are given in terms of $E_{R}$, which is finally fitted to the data once the finite $D^{* 0}$ width is restored in the definition of the three-momentum, cf. Eq. (37).

The formulas derived to actually fix $\beta, \lambda$ and $M_{\mathrm{CDD}}$ are given in Appendix C, Eqs. (C.3), (C.4), (C.7) and (C.8). There it is shown that indeed one has two solutions, that we indicate by case 3.I (first solution) and case 3.II (second solution). The expressions simplify in the limit $\left|E_{R}\right| / \Delta \rightarrow 0$, which is relevant for $X$ (3872) given its small energy, and the two solutions coalesce in just one. In this case we show that there are two poles in different RS's, confirming the intuitive physical reasons given at the beginning of this section.

The values for the fitted parameters are given in Eq. (65) for case 3.I and in Eq. (66) for case 3.II. The resulting event distributions are shown by the blue dash-double-dotted (case 3.I) and green dash-dotted (case 3.II) lines in Fig. 4 and in the histogram of Fig. 5. These lines are hardly distinguish-

\footnotetext{
11 The other two equations give the values for the CDD pole positions in the two isospin limits considered. This is information which is of no use.
}

able among them and can only be differentiated with respect to case 1 in the $D^{0} \bar{D}^{* 0}$ event distribution, as one can appreciate clearly from Fig. 5. With respect to cases 2.I-II we have the already commented shift of the peak in the $J / \psi \pi^{+} \pi^{-}$ event distributions, more clearly seen in Fig. 9. The global reproduction of data is of similar quality as the one already achieved by the pure bound-state and virtual-state cases. The values for the pole positions and CDD parameters are given in Tables 2 and 3, respectively.

For case 3.I the resulting pole position for the bound state (the pole in the first RS) is $-0.50_{-0.03}^{+0.04} \mathrm{MeV}$, while the pole position for the virtual state (the pole in the second RS) is $-0.68_{-0.03}^{+0.05} \mathrm{MeV}$. In both cases there is a tiny imaginary part of the order of $10^{-3} \mathrm{MeV}$ which is beyond the precision shown. The compositeness of the $D^{0} \bar{D}^{* 0}$ state in the bound state, evaluated in the same way as explained at the end of Sect. 6.1, is 0.06 , i.e., the $D^{0} \bar{D}^{* 0}$ component only constitutes around a $6 \%$ of the $X(3872)$ due to the extreme proximity of the CDD pole to the $D^{0} \bar{D}^{* 0}$ threshold. As shown in Table 3 the CDD pole is much closer to threshold than the bound-state pole. As a result, other components are dominant, e.g. one could think of the conventional $\chi_{c 1}(2 P)$ as $c \bar{c}$, tetraquarks, hybrids, etc. $[4,33,51-56]$. These facts about the smallness of the imaginary part of the two near-threshold poles and the small compositeness for the bound state can be understood in algebraic terms in the limit $\left|E_{R}\right| / \Delta \rightarrow 0$ as shown in Appendix C; cf. Eqs. (C.18), (C.21) and (C.24). Indeed, they are related because if the $X(3872)$ has such a small value for the compositeness, then it is fairly insensitive to the width of $D^{* 0}$. In addition, one also has a deep virtual state located at $E_{3} \approx-\beta^{2} / 2 \mu-i \Gamma_{*} / 2$, that is quite insensitive to the CDD pole contribution, which is strongly suppressed at those energies as explained in more detail after Eq. (C.23).

Let us notice that the ERE for the present near-threshold bound state fails because ERE is not applicable since the zero is closer to threshold than the pole. Taking $\Gamma_{*}=0$ and calculating $a$ and $r$ we obtain the central values (errors are given in Table 3)

$a=0.27 \mathrm{fm} \rightarrow 0, \quad r=-847.8 \mathrm{fm} \rightarrow \infty$,

while the binding momentum is $\kappa=\sqrt{2 \mu\left|E_{X}\right|}=31.0 \mathrm{MeV}$ $<<1 /|a|=723 \mathrm{MeV}$. The pole position in the first (second) RS that stems from the ERE up to the effective range is -0.17 $(-0.18) \mathrm{MeV}$, which is indeed very different from the actual pole position of the bound (virtual) state in the full amplitude at $-0.50(-0.68) \mathrm{MeV}$.

For the second solution, i.e. case 3.II, we have much larger values of $\lambda$ and $M_{\mathrm{CDD}}$ than for the first one, compare between the last two rows in Table 3. This is a common characteristic to any value $E_{R}<0$ as shown in Fig. 8, where the values of $\lambda$ (left panel) and $M_{\mathrm{CDD}}$ (right panel) are given as a function of $E_{R}$ for the first (black solid) and second (red dashed lines) solutions. 

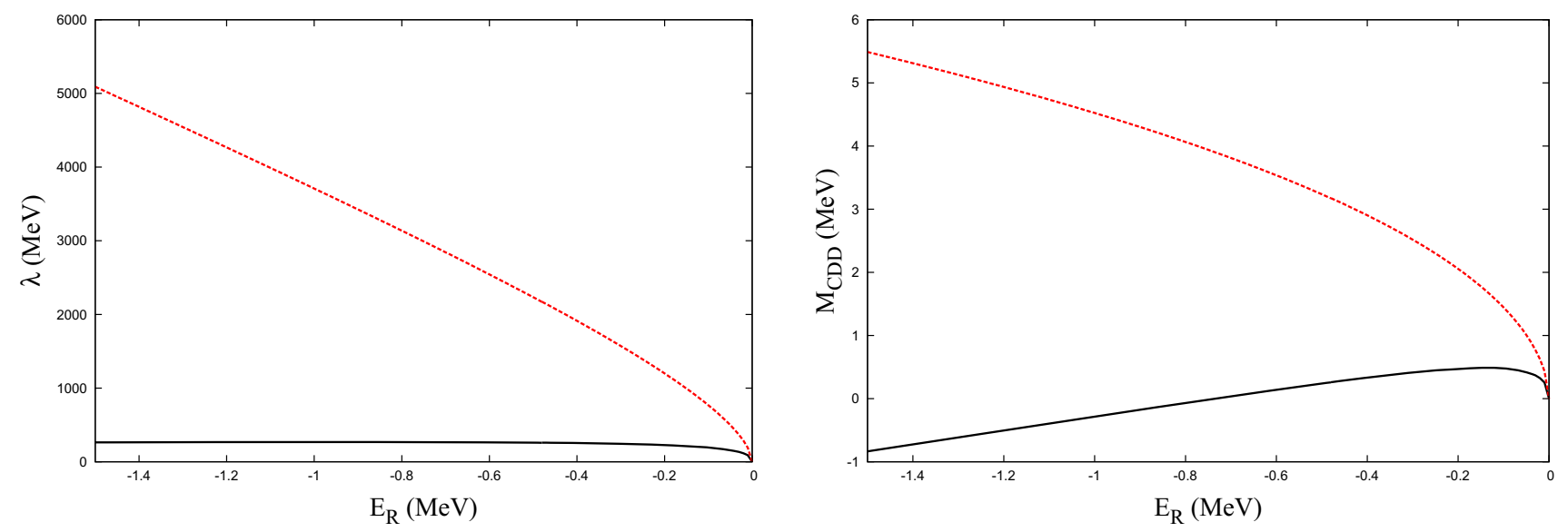

Fig. 8 The left panels shows $\lambda$ and the right one $M_{\mathrm{CDD}}$ as a function of $E_{R}$ for case 3.I (black solid) and case 3.II (red dashed lines)

The pole positions in the first and second RS's are given in the second column of the last line of Table 2. The fact that for this second solution $M_{\mathrm{CDD}}$ is further away from threshold than for the solution case 3.I is an indication that compositeness is larger for the former than for the latter. For case 3.II we obtain now $X=0.16$, while before it was around 0.06 . This is in agreement with our expectations, but still $X$ is small and the state is dominantly a bare (non-molecular) one. ${ }^{12}$ The residues for this case are also given in column four of Table 2. They are larger by around a factor 3 compared to the first solution, which is in line with the increase in the value of compositeness. The bound states for cases 3.I-II have a normalization integral $\mathcal{N}=1$ so that it is legitimate to interpret the $Y_{F}$ as yields.

The ERE expansion for case 3.II is better behaved because $M_{\mathrm{CDD}}$ is relatively further from threshold. We now have the values $\left(\Gamma_{*}=0\right.$ should be understood in the following discussions)

$a=+1.57_{-0.02}^{+0.05} \mathrm{fm}$

$r=-43.75_{-0.61}^{+0.24} \mathrm{fm}$,

where $r$ is still much larger than a typical range of strong interactions and $a$ is much smaller than $1 / \sqrt{2 \mu\left|E_{X}\right|}$. These facts just reflect the dominant bare nature of $X(3872)$ in this case. The ERE up to $r$ gives rise to a bound state located at $-0.45 \mathrm{MeV}$, already very close to the full-solution result at $-0.51 \mathrm{MeV}$, which is much better than for case 3.I. Regarding the virtual-state pole the ERE also produces a pole in the second RS at $-0.76 \mathrm{MeV}$, while the full result is at $-1.06 \mathrm{MeV}$, around a $25 \%$ of error. This worse behavior

\footnotetext{
12 Taking the same value for the residue of $D^{+} D^{*-}$ as for $D^{0} \bar{D}^{* 0}$, because of isospin symmetry, we can evaluate straightforwardly the compositeness of the $D^{+} D^{*-}$ channel and is a factor 3 smaller than for $D^{0} \bar{D}^{* 0}$. Then, summing it to 0.15 , we end with 0.20 as an estimated value for the total compositeness of the $D \bar{D}^{*}$ states, which is still small. For case 3.I it would be around only 0.05 .
}

of the ERE to determine the location of the virtual-state pole is to be expected because the radius of convergence of the ERE is $2 \mu M_{\mathrm{CDD}}$, and the virtual-state pole is closer to this limit than the bound-state one. More contributions are certainly needed as the ERE is applied to energies that are closer to the radius of convergence of the expansion.

Related to this discussion we consider the Weinberg's compositeness theorem for a near-threshold bound state, which reads [10]

$$
\begin{aligned}
a & =\frac{2(1-Z)}{(2-Z) \kappa}, \\
r & =-\frac{Z}{(1-Z) \kappa},
\end{aligned}
$$

where $Z$ is the elementariness, or $1-X$. This criterion, as discussed in the Introduction, cannot be applied if a CDD is closer to threshold than the bound-state pole, as it happens for case 3.I, because it relies on the applicability of the ERE up to the effective range. For case 3.II this is not the case, but still the CDD pole is quite close so that energy dependences beyond the effective range play a role. The Weinberg's compositeness relation gives $Z_{a}=0.86$ and $Z_{r}=0.87$, when using $a$ and $r$ to calculate it from the first and second expressions in Eq. (84), respectively. These numbers compare very well with $Z=1-X=0.84$, where $X=0.16$ is determined above and given in Table 2. These values of elementariness so close to 1 for cases 3.I-II are also in agreement with the expectation of having two poles close to threshold in adjacent RS's (virtual- and bound-state poles simultaneously), which fits very well within Morgan's criterion for a preexisting or non-molecular state [57].

Our fit for case 3.II corresponds to the following central values for the parameters characterizing the scattering model of Ref. [11], in terms of the exchange of a bare state and direct scattering between $D^{0} \bar{D}^{* 0}: g_{f 0}=0.014, \gamma_{V}=-561.7$ and $E_{f}=-0.71 \mathrm{MeV}$. Compared with cases 2.I-II one observes 
a significant smaller value for $g_{f 0}$ and $\left|E_{f}\right|$. The resulting pole trajectories as $g_{f}$ is increased from $0.1 g_{f 0}$ up to $20 g_{f 0}$, with $E_{V}$ and $\gamma_{V}$ held fixed, are shown in the bottom-right panel of Fig. 6, with a similar behavior for case 3.I, which is not shown. Notice that because $E_{f}<0$ one has in the decoupling limit $\left(g_{f} \rightarrow 0\right)$ a bound- and a virtual-state pole at $\pm i \sqrt{2 \mu\left|E_{f}\right|}=33.2 \mathrm{MeV}$. This type of pole movement as a strength parameter varies is different from those discussed in Ref. [45], because the near-threshold poles do not belong to the trajectory of two complex poles associated with the same resonance. However, this is the case for the two virtual state poles, the shallow and deep ones, as clearly seen in the figure.

The poles trajectories in the last panel of Fig. 6 do not belong either to the ones discussed explicitly in Ref. [11], where much smaller values of $\left|\gamma_{V}\right|$ are considered. The reason behind is the misused performed in this reference of the relationship between the pole positions $k_{i}, i=1,2,3$ and the position of the CDD pole ${ }^{13}$

$M_{\mathrm{CDD}}=-\frac{1}{2 \mu}\left(k_{3}\left(k_{1}+k_{2}\right)+k_{1} k_{2}\right)$.

The point is that Ref. [11] concluded from this equation that it is necessary that the three poles be shallow ones $\left(\left|k_{i}\right| \ll \Delta\right)$ in order to have a near-threshold CDD pole $\left(\left|M_{\mathrm{CDD}}\right| \ll \Delta\right)$. However, this conclusion is just sufficient but not necessary. The other possibility is that $k_{1}$ and $k_{2}$ nearly cancel each other (such that $\left|k_{1}+k_{2}\right|=\mathcal{O}\left(\left|k_{1,2} / k_{3}\right|^{2}\right.$ ), without being necessary that $\left|k_{3}\right| \ll \Delta$ (which in our case is given by $\beta \gg \Delta$ ). This is what happens particularly for case 3.I with $k_{1}=i 31.0$ and $k_{2}=-i 36.2 \mathrm{MeV}$, so that the CDD pole is almost on top of threshold. Therefore, one does not really need that three poles lie very close to threshold to end with a shallow CDD pole.

It is also interesting to apply the spectral density introduced in Eq. (74) to evaluate the compositeness and elementariness of the bound states in cases 3.I-II. For such a purpose one has to integrate the spectral density up to infinity with $W$ defined as

$W=\int_{0}^{\infty} \mathrm{d} E \omega(E)$,

and interpreted as the compositeness $X$ [7]. The normalization to 1 of the bare state then guarantees that $Z=1-X$, which provides us with the elementariness. Notice that this is the third way that we have introduced to evaluate the compositeness of a bound state. Namely, we can evaluate it in terms of the residue of $t(E)$ at the pole position, Eq. (67), Weinberg's relations, Eq. (84), or in terms of the spectral density,

\footnotetext{
13 Similarly, one can also derive the equalities $\lambda=-i\left(k_{3}^{2}\left(k_{1}+k_{2}\right)+\right.$ $\left.k_{3}\left(k_{1}+k_{2}\right)^{2}+k_{1} k_{2}\left(k_{1}+k_{2}\right)\right) / 2 \mu$ and $\beta=i \sum_{i} k_{i}$. These relations can easily be worked out from the secular equation which is a third-order polynomial.
}

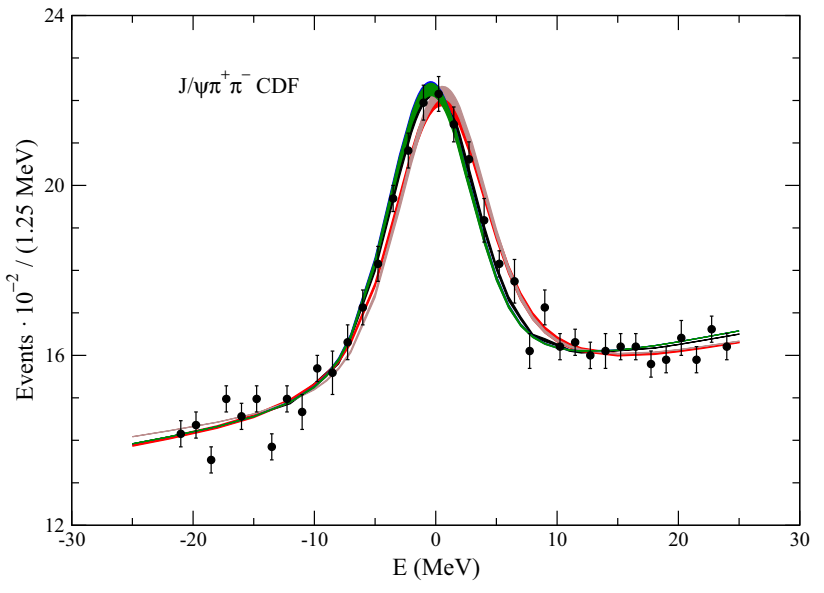

Fig. 9 The curves of the different cases for the $J / \psi \pi^{+} \pi^{-}$event distribution from the inclusive $p \bar{p}$ scattering [18] are given with error bands included. The black-, red-, brown-, blue-, and green-filled bands correspond to cases 1, 2.I, 2.II, 3.I and 3.II, in order

Eq. (86). The latter also provides remarkably close values to the previous ones so that for case 3.II one has $W=0.16$, while for case 3.I (in which case Weinberg's result does not apply) one obtains $W=0.06$.

We have also checked that our results are stable if the $D^{+} D^{*-}$ channel is explicitly included in $d(E)$ by using the same formalism as in Ref. [31]. At the practical level this amounts to modifying the denominator of $d(E)$ such that $\beta \rightarrow \beta-i\left[k^{(2)}(E)-\bar{k}^{(2)}(0)\right]$, with $k^{(2)}(E)$ the $D^{+} D^{*-}$ three-momentum given by the expression $k^{(2)}(E)=$ $\sqrt{2 \mu_{2}\left(E+i \widetilde{\Gamma}_{*} / 2-\Delta\right)}$, where $\mu_{2}$ is the reduced mass and $\widetilde{\Gamma}_{*}$ the width of the $D^{*+}$ resonance [19], while $\bar{k}^{(2)}(0)$ is given by the same expression with $E+i \widetilde{\Gamma_{*}} / 2 \rightarrow 0$. For example, by redoing the fit in this case the fitted parameters match very well the values in Eq. (65) within errors.

In order to have an extra perception on how the uncertainty in the fitted parameters influences our results, we also show in Fig. 9 the error bands of the curves obtained from the different cases considered for the reproduction of the CDF data on the inclusive $p \bar{p}$ scattering to $J / \psi \pi^{+} \pi^{-}$[18]. We have not shown the error bands for the other data, and just shown the curves obtained from the central values of the fit parameters in Fig. 4, because the typical width for every error band in each line is of similar size as the one shown in Fig. 9. We have chosen this data because it is the one with the smallest relative errors, having the largest statistics and smallest bin width. In addition, the curves are so close to each other that in the scale of the Fig. 4 it would be nearly impossible to distinguish between all the curves with additional error bands included. Here we offer just one panel which also allows us to use a larger size for it and be able to distinguish better between lines with error bands. But even then one clearly sees in Fig. 9 that the bands for cases 1 and 3.I-II mostly 
overlap each other so that they are hardly distinguishable. The cases 2.I-II can be differentiated from the rest because there is a slight shift of the peak structure to the right. However, this shift becomes smaller and all the bands overlap each other if we had excluded in the fit the $D^{0} \bar{D}^{* 0}$ event distribution of the BaBar Collaboration [15].

\section{Conclusions}

Since its exciting discovery [58] $X(3872)$ has been extensively studied, for a recent review see Ref. [59]. Among the many theoretical approaches [1-6,33,51-56,60-63], we have paid special attention to the applicability of the popular ERE approximation up to and including the effective-range contribution to study near-threshold states like $X$ (3872). We have elaborated about the fact that the ERE convergence radius might be severely limited due to the presence of nearthreshold zeros of the partial wave, the so-called CastillejoDalitz-Dyson poles. We have then derived a parameterization that is more general than the ERE up to and including effective range, ${ }^{14}$ but it can deal as well with the presence of a CDD pole arbitrarily close to threshold. We have shown too that other parameterizations based on the picture of the exchange of a bare state plus direct interactions between the $D^{0} \bar{D}^{* 0}$ can also be matched into our parameterization $[11,12]$. In particular, Ref. [11] already stressed the strong impact that a possible near-threshold zero would have in the $D^{0} \bar{D}^{* 0} S$-wave amplitude. However, we have shown that the conclusion there stated about the necessity of three simultaneous shallow poles to end with a near-threshold zero is sufficient but not necessary, because it is just enough having two such poles. We have illustrated this conclusion with a possible scenario for $X(3872)$ in which there are only two nearthreshold poles, a bound state in the first RS and a virtual-state one in the second RS.

We have then reproduced several event distributions around the $D^{0} \bar{D}^{* 0}$ threshold including those of $D^{0} \bar{D}^{* 0}$ and $J / \psi \pi^{+} \pi^{-}$from charged $B$ decays measured by the BaBar and Belle Collaborations and the higher-statistics CDF $J / \psi \pi^{+} \pi^{-}$event distributions from inclusive $p \bar{p}$ scattering at $\sqrt{s}=1.96 \mathrm{TeV}$. Our formalism has as limiting cases those of Refs. [1,3], but it can also include other cases in which the presence of a CDD pole plays an important role. In this respect we are able to find other interesting scenarios beyond those found in Refs. [1,3] that can reproduce data fairly well, and without increasing the number of free parameters. In two of these new situations $X$ (3872) is simultaneously a bound and a virtual state, while in others the $X(3872)$ is a double or a triplet virtual-state pole. In the limit

\footnotetext{
14 Indeed up to the next shape parameter, $v_{2}$. Let us recall again that the ERE is more general than a Flatté parameterization.
}

of vanishing width of $D^{* 0}$ these poles become degenerate and produce a higher-order pole (of second or third order). Thus, our parameterization constitutes in the latter cases a simple example for higher-order $S$-matrix poles that could have a clear impact on particle physics phenomenology.

In this respect, we stress that the corrections to the pole position when taking into account the finite width of the $D^{* 0}$ resonance, $\Gamma_{*}$, are non-analytic for the higher-order poles of order $n>1$. In such situations one has that the leading corrections are proportional to $\rho^{1 / n}$, with $\rho=\Gamma_{*} /\left|E_{R}\right|$, being $E_{R}$ the real part of the pole position with respect to the $D^{0} \bar{D}^{* 0}$ threshold without the $D^{* 0}$ width. This could be an important source of $D^{0} \bar{D}^{* 0}$ partial width for the $X(3872)$. Indeed, with this mechanism the absolute value of twice the imaginary part of the pole positions for the triplet-pole scenario could be nearly as large as $1 \mathrm{MeV}$, despite $\Gamma_{*}$ being only around $0.065 \mathrm{keV}[2,19]$. Thus a measurement of the total width of $X$ (3872) might be useful to discriminate between the discussed scenarios.

Further, while the compositeness is equal to 1 for the bound-state case analyzed making use of the ERE including only the scattering length [3], it is nearly zero for cases 3.I-II in which $X(3872)$ is a simultaneous virtual and bound state. Case 3.I has the closest CDD pole to threshold, even closer than the pole positions. In this respect, we also estimate that the $X(3872)$ is mostly $D^{0} \bar{D}^{* 0}$ for the double virtualstate case, because the CDD pole is relatively far away from threshold, while in the triplet-pole case the elementariness is dominant as indicated by the closeness of the CDD to threshold. We have verified quantitative these conclusions as well by employing the spectral density function.

From another perspective, we have shown that using a more refined treatment of $D^{0} \bar{D}^{* 0}$ scattering $X(3872)$ can be a bound-state, a double/triplet virtual-state pole or two types of simultaneous virtual and bound states with poles occurring in both the physical and unphysical sheets, respectively. All these scenarios can give a rather acceptable reproduction of the experimentally measured event distributions. Up to some extent this situation recalls the case of $X(1835)$, for which the energy-dependent $J / \psi \rightarrow \gamma p \bar{p}$ event distribution is nicely reproduced by purely final-state interactions of $p \bar{p}$ [64]. However, this treatment fails to describe the data when a more elaborate model is taken. Only the generation of a $p \bar{p}$ bound state in the scattering amplitude is able to reproduce the data within this more sophisticated model [65-67].

From our present results and this experience, more efforts are still needed to finally unveil the nature of the acclaimed $X$ (3872), which is the first $X Y Z$ state observed. In this respect, we mention that there are visible differences between the different scenarios analyzed in the $D^{0} \bar{D}^{* 0}$ invariant mass distributions in the peak of $X(3872)$, as shown in detail in Fig. 5, in particular between the scenarios I, 2.II and the rest. 
We have to indicate that the present data shows a clear displacement towards higher masses of the $X$ (3872) peak in the BaBar Collaboration data [15] as compared with the Belle Collaboration one [17]. Indeed, if the former data is excluded in the fits the shift towards the right of the signal peak for cases 2.I-II in the $J / \psi \pi^{+} \pi^{-}$CDF data [18], cf. Fig. 9, diminishes considerably. Thus, a future high-statistic experiment on $B \rightarrow K D^{0} \bar{D}^{* 0}$ might be very helpful to differentiate between different cases, if complemented with highprecision data on $J / \Psi \pi^{+} \pi^{-}$. Another way to discriminate between different possibilities might be the measurement of the partial-decay width of $X(3872)$ to $D^{0} \bar{D}^{* 0}$, as mentioned above. Lattice QCD can also provide interesting information from where one could deduce the $D^{0} \bar{D}^{0 *}$ near-threshold scattering amplitude and then determine whether there is a CDD pole or not. Indeed, present Lattice $\mathrm{QCD}$ results point towards the importance of the interplay between quark and meson degrees of freedom to generate $X$ (3872) [68-70]. Another interesting idea was put forward by Voloshin in Ref. [21], indicating the convenience to measure the $D^{0} \bar{D}^{* 0} \pi^{0}$ Dalitz plot to distinguish between the molecular and quarkonium picture for $X(3872)$.

Acknowledgements We would like to thank C. Hanhart for inspiring and compelling discussions. Interesting discussions are also acknowledged to Q. Zhao. This work is supported in part by the MINECO (Spain) and ERDF (European Commission) Grant FPA2013-40483-P and the Spanish Excellence Network on Hadronic Physics FIS201457026-REDT.

Open Access This article is distributed under the terms of the Creative Commons Attribution 4.0 International License (http://creativecomm ons.org/licenses/by/4.0/), which permits unrestricted use, distribution, and reproduction in any medium, provided you give appropriate credit to the original author(s) and the source, provide a link to the Creative Commons license, and indicate if changes were made.

Funded by SCOAP ${ }^{3}$.

\section{Appendix A: Obtaining properly normalized yields}

In order to fit the event distributions when using $d(E)$ with a CDD included, the evaluation of $\alpha$ entering in Eq. (51) for calculating the event distributions requires one to work out the pole position, cf. Eq. (43), which is not easily expressed in terms of free parameters. This is not the case when using the function $f(E)$ because in this case $\alpha$, Eq. (45), is given directly in terms of $\gamma$, a fit parameter. Thus, when $d(E)$ is employed we first perform the fits such that $|\alpha|^{2}$ is reabsorbed in the normalization constants multiplying the signal contribution. In this way one avoids having to calculate the pole position for each iteration in the fit procedure. To be specific, we use the following expression for the fits to the $D^{0} \bar{D}^{* 0}$ event distributions:

$$
\begin{aligned}
& N_{i}\left(E_{i}\right)=\int_{E_{i}-\Delta / 2}^{E_{i}+\Delta / 2} \mathrm{~d} E^{\prime \prime} \int_{0}^{\infty} \mathrm{d} E^{\prime} R\left(E^{\prime \prime}, E^{\prime}\right) \sqrt{E^{\prime}} \\
& \quad \times\left\{\mathfrak{b}_{D} \int_{-\infty}^{\infty} \mathrm{d} E|d(E)|^{2} \frac{\Gamma_{*}}{\left(E^{\prime}-E\right)^{2}+\Gamma_{*}^{2} / 4}+\widetilde{\operatorname{cbg}}_{D}\right\} .
\end{aligned}
$$

For the fits to the $J / \psi \pi^{+} \pi^{-}$event distributions, we employ

$$
\begin{gathered}
N_{i}\left(E_{i}\right)=\vartheta_{J}\left\{\mathfrak{b}_{J} \int_{E_{i}-\Delta / 2}^{E_{i}+\Delta / 2} \mathrm{~d} E^{\prime} \int_{-\infty}^{\infty} \mathrm{d} E R\left(E^{\prime}, E\right)|d(E)|^{2}\right. \\
\left.+\widetilde{\operatorname{cbg}}_{J} \Delta\right\},
\end{gathered}
$$

where $\vartheta_{J, D}=1$ for BaBar data and for Belle it corresponds to the ratio of the number of $B \bar{B}$ pairs produced in Belle and BaBar for each type of $B^{+} \rightarrow J / \psi \pi^{+} \pi^{-}$decays, namely, $N_{B \bar{B}}^{\mathrm{Belle}} / N_{B \bar{B}}^{\mathrm{BaBar}}$. The number of $B \bar{B}$ pairs is given in Table 1 . Once the fit is performed we can deduce the values of the "yields" $Y_{D}$ and $Y_{J}$ (here the quotation marks are introduced because it is required that $\mathcal{N} \simeq 1$, with the normalization constant $\mathcal{N}$ introduced in Eq. (52), in order to interpret meaningfully these constants as yields). The appropriate relations can be deduced by comparing Eqs. (59) and (54) with Eqs. (A.1) and (A.2), in order. They read

$$
\begin{aligned}
Y_{D} & =\mathfrak{b}_{D} \frac{\sqrt{2} \pi \sqrt{E_{X}+\sqrt{E_{X}^{2}+\Gamma_{*}^{2} / 4}}}{g_{\mathrm{CDD}}}, \\
Y_{J} & =\frac{\mathfrak{b}_{J}}{g_{\mathrm{CDD}}}, \\
g_{\mathrm{CDD}} & =\frac{\Gamma_{X}}{2 \pi|\alpha|^{2}} .
\end{aligned}
$$

The pole position $E_{X}-i \Gamma_{X} / 2$ and associated momentum $k_{P}$ are determined from the fitted values of the parameters. We also have the trivial relations between the background parameters

$$
\begin{aligned}
\widetilde{\operatorname{cbg}}_{J} & =N_{B \bar{B} ; J}^{\mathrm{BaBar}} \operatorname{cbg}_{J}, \\
\widetilde{\operatorname{cbg}}_{D} & =N_{B \bar{B} ; D}^{\mathrm{BaBar}} \operatorname{cbg}_{D} .
\end{aligned}
$$

An analogous procedure is also applied when fitting the $J / \psi \pi^{+} \pi^{-}$event distribution from the inclusive $p \bar{p}$ scattering measured by the CDF Collaboration [18]. In this way, we can extract $Y_{J}^{(p)}$ by applying Eq. (A.3) too.

\section{Appendix B: Pole positions with finite $\Gamma_{*}$ in cases 2.I, II}

For $\Gamma_{*} \neq 0$ the relation between energy and momentum is $E=k^{2} / 2 \mu-i \Gamma_{*} / 2$, cf. Eq. (37), and the secular equation (71) becomes

$\lambda+\left(\frac{k^{2}}{2 \mu}-i \frac{\Gamma_{*}}{2}-M_{\mathrm{CDD}}\right)(-i k+\beta)=0$. 
After some simplifications it can be written as

$$
\begin{aligned}
& (k+i \varkappa)^{2}\left(k-i \frac{\varkappa^{2}-2 \mu M_{\mathrm{CDD}}}{2 \varkappa}\right) \\
& -i \mu \Gamma_{*}\left(k+i\left[\frac{3 \varkappa}{2}+\frac{\mu M_{\mathrm{CDD}}}{\varkappa}\right]\right)=0,
\end{aligned}
$$

with $\varkappa=\sqrt{2 \mu\left|E_{R}\right|}$.

For case 2.I $\left|M_{\mathrm{CDD}}\right| \gg\left|E_{R}\right|$ and the previous equation becomes in good approximation

$(k+i \beta)\left((k+i \varkappa)^{2}-i \mu \Gamma_{*}\right)=0$.

Its solution gives rise to the pole positions in the $k$ plane given in Eq. (77) and in the $E$ plane are those of Eq. (78).

For case 2.II, $M_{\mathrm{CDD}}=-3 E_{R}$ and Eq. (B.2) becomes

$(k+i \varkappa)^{3}-i \mu \Gamma_{*}(k+i 3 \varkappa)=0$.

In terms of the dimensionless variable

$t=\frac{k+i \varkappa}{\varkappa}$

Eq. (B.4) reads

$t\left(t^{2}-i \frac{\Gamma_{*}}{2\left|E_{R}\right|}\right)=-\rho$.

This equation can be solved in a power expansion of $\rho$ with the leading result

$$
\begin{aligned}
& t_{1}=-\rho^{\frac{1}{3}}+\mathcal{O}\left(\rho^{\frac{2}{3}}\right), \\
& t_{2}=e^{-i \frac{\pi}{3}} \rho^{\frac{1}{3}}+\mathcal{O}\left(\rho^{\frac{2}{3}}\right), \\
& t_{3}=e^{i \frac{\pi}{3}} \rho^{\frac{1}{3}}+\mathcal{O}\left(\rho^{\frac{2}{3}}\right) .
\end{aligned}
$$

In the momentum and energy variables this solution gives rise to Eqs. (79) and (80), in order.

\section{Appendix C: Formalism for cases 3.I-II: simultaneous virtual- and bound-state poles}

Let us connect with the $T$-matrix derived in a previous paper by one of the authors in which the resonance $\Lambda_{c}(2535)^{+}$ was studied [31]. This is a resonance that also lies very close to the $\pi \Sigma_{c}$ thresholds. Namely, it has a small width of $2.6 \pm 0.6 \mathrm{MeV}$ [19] and its mass is $4.37 \mathrm{MeV}$ above the $\pi^{0} \Sigma_{c}^{+}$threshold, and 1.06 and $1.30 \mathrm{MeV}$ below the nearly degenerate thresholds of $\pi^{+} \Sigma_{c}^{0}$ and $\pi^{-} \Sigma_{c}^{++}$, respectively. Reference [31] explored the viability of this resonance to be a preexisting one, with $Z \simeq 1$, so that its actual pole position is unaffected by taking as masses in the isospin limit those in the $\pi^{0} \Sigma_{c}^{+}$or in the $\pi^{-} \Sigma_{c}^{++}$states. ${ }^{15}$

15 Contrarily to the more general expectations of Ref. [31] the parameter $\lambda$ could depend strongly on the isospin mass taken.
Here we have adopted a similar point of view and required the invariance of the virtual-state pole position independently of whether one takes in the isospin limit the masses of the neutral mesons $D^{0}, D^{* 0}$ or of the charged ones $D^{+}, D^{*+}$. We denote the channel $D^{0} \bar{D}^{* 0}$ by 1 and the channel made by the charged particles $D^{+} D^{*-}$ by 2 . In the isospin limit, we replace $\lambda$ by $\tilde{\lambda}$ in Eq. (36) with

$\lambda \rightarrow 2 \tilde{\lambda}$

because of an isospin Clebsch-Gordan coefficient squared to combine $D \bar{D}^{*}$ in isospin 0 . Specifically, in the isospin limit we use the scattering amplitude

$t_{j}(\sqrt{s})=\left(\frac{\tilde{\lambda}}{\sqrt{s}-M_{\mathrm{CDD}}^{(j)}}+\beta_{j}-i k^{(j)}(\sqrt{s})\right)^{-1}$,

where $s$ is the usual Mandelstam variable and the subscript in $t_{j}(\sqrt{s})$ refers to take the isospin limit with the masses of the state $j$. For each case the CDD pole position is indicated by $M_{\mathrm{CDD}}^{(j)}$, and $k^{(j)}(\sqrt{s})=\sqrt{2 \mu_{j}\left(\sqrt{s}-\sigma_{j}\right)}$, with $\mu_{j}$ the reduced mass and $\sigma_{j}$ the threshold mass of the $j$ th $D \bar{D}^{*}$ state. In addition, following Ref. [31], we have

$$
\begin{aligned}
& \beta_{j}=8 \pi \sigma_{j} \alpha+\rho_{j}, \\
& \rho_{j}=\frac{1}{\pi}\left(M_{D}^{(j)} \log \frac{M_{D}^{(j)}}{M_{\pi^{+}}}+M_{D^{*}}^{(j)} \log \frac{M_{D^{*}}^{(j)}}{M_{\pi^{+}}}\right) .
\end{aligned}
$$

The previous equation results by taking the non-relativistic reduction of the unitarity loop function for the $s$-channel intermediate state. We have also used quite an obvious notation for the masses involved. The real and imaginary parts of the momentum at the resonance in the second RS are denoted by $k_{r}^{(j)}$ and $-k_{i}^{(j)}$ and can be calculated from $k_{r}^{(j)}-i k_{i}^{(j)} \equiv-\sqrt{2 \mu_{j}\left(M_{R}-i \Gamma_{R} / 2-\sigma_{j}\right)}$, with the argument of the radicand taken between $[0,2 \pi$ [. The expressions for $\tilde{\lambda}, \alpha$ and $M_{\mathrm{CDD}}^{(j)}$ that result by imposing that each $t_{j}(\sqrt{s})$ has a pole in the second RS at $M_{R}-i \Gamma_{R} / 2$, with $M_{R}=M_{D^{0}}+M_{D^{* 0}}+E_{R}$ fixed can be found in Ref. [31]. We write them here as

$$
\begin{aligned}
\alpha= & \frac{\Gamma_{R}\left(k_{i}^{(1)}-\rho_{1}\right)+2 k_{r}^{(1)}\left(M_{\mathrm{CDD}}^{(1)}-M_{R}\right)}{8 \pi \sigma_{2} \Gamma_{R}}, \\
\tilde{\lambda}= & \frac{k_{r}^{(1)}\left[\left(M_{\mathrm{CDD}}^{(1)}-M_{R}\right)^{2}+\Gamma_{R}^{2} / 4\right]}{\Gamma_{R} / 2}, \\
M_{\mathrm{CDD}}^{(1)}= & \frac{\chi_{11} \pm \sqrt{\chi_{12}}}{k_{r}^{(1)}\left(k_{r}^{(1)} \sigma_{2}^{2}-k_{r}^{(2)} \sigma_{1}^{2}\right)}, \\
\chi_{21}= & M_{R} k_{r}^{(1)}\left(k_{r}^{(1)} \sigma_{2}^{2}-k_{r}^{(2)} \sigma_{1}^{2}\right)+\Gamma_{R} k_{r}^{(1)} \sigma_{2}\left(-k_{i}^{(1)} \sigma_{2}\right. \\
& \left.+\rho_{1} \sigma_{2}+k_{i}^{(2)} \sigma_{1}-\rho_{2} \sigma_{1}\right) / 2,
\end{aligned}
$$




$$
\begin{aligned}
\chi_{12}= & \frac{\Gamma_{R}^{2}}{4} k_{r}^{(1)} k_{r}^{(2)} \sigma_{1}^{2}\left\{\sigma_{2}^{2}\left[k_{r}^{(1)}\left(k_{r}^{(1)}-k_{r}^{(2)}\right)+\left(k_{i}^{(1)}-\rho_{1}\right)^{2}\right]\right. \\
& -2 \sigma_{1} \sigma_{2}\left(k_{i}^{(2)}-\rho_{2}\right)\left(k_{i}^{(1)}-\rho_{1}\right) \\
& \left.+\sigma_{1}^{2}\left[k_{r}^{(2)}\left(k_{r}^{(2)}-k_{r}^{(1)}\right)+\left(k_{i}^{(2)}-\rho_{2}\right)^{2}\right]\right\}, \quad \text { (C.4) }
\end{aligned}
$$

These equations provide us with two different solutions, which stem from the \pm sign in the expression for $M_{\mathrm{CDD}}^{(1)}$. We refer to them as the first and second solutions.

In the limit $\Gamma_{R} \rightarrow 0^{+}$we end with similar expressions for $\tilde{\lambda}$ and $\beta_{1}$ as Eq. (70),

$$
\begin{aligned}
\tilde{\lambda} & =\frac{\mu_{1}}{\varkappa_{1}}\left(M_{\mathrm{CDD}}^{(1)}-E_{R}\right)^{2}, \\
\beta_{1} & =\frac{\mu_{1}}{\varkappa_{1}}\left(M_{\mathrm{CDD}}^{(1)}-3 E_{R}\right) \rightarrow \alpha=\frac{\beta_{1}}{8 \pi \sigma_{1}}-\rho_{1},
\end{aligned}
$$

with $\varkappa_{1}=\sqrt{2 \mu_{1}\left|E_{R}\right|}$.

The coupled-channel $S$-wave amplitude for channels 1 and 2, using again the correspondingly adapted expression of Ref. [31], reads

$t(\sqrt{s})=\left(\frac{2 \tilde{\lambda}}{\sqrt{s}-\widetilde{M}_{\mathrm{CDD}}}+\beta_{1}+\beta_{2}-i k^{(2)}(\sqrt{s})-i k^{(1)}(\sqrt{s})\right)^{-1}$.

In this formula one implicitly assumes that the main isospin breaking corrections between the different coupled channels are expected to arise from the dependence of the three-momenta $k^{(i)}$ on their threshold because of the associated branch point singularity at each nearby threshold [31].

The parameters $\tilde{\lambda}, \beta_{1}$ and $\beta_{2}$, cf. Eq. (C.3), are fixed here from Eq. (C.4) in terms of $E_{R}$. We still have to determine $\widetilde{M}_{\mathrm{CDD}}$, which is fixed by requiring that $t(\sqrt{s})$ have a boundstate pole (in the first RS) at $\sqrt{s}=M_{R}$,

$\tilde{M}_{\mathrm{CDD}}=M_{R}-2 \tilde{\lambda} /\left(\beta_{1}+\beta_{2}-i k^{(2)}\left(M_{R}\right)-i k^{(1)}\left(M_{R}\right)\right)$.

In this way, the parameters to be employed in Eq. (36) for case 3 introduced in Sect. 6.3 are:

$$
\begin{aligned}
\lambda & =2 \tilde{\lambda}, \\
M_{\mathrm{CDD}} & =\widetilde{M}_{\mathrm{CDD}}-\sigma_{1}, \\
\beta & =\beta_{1}+\beta_{2}-i k^{(2)}\left(\sigma_{1}\right) .
\end{aligned}
$$

Notice that the three-momentum of the channel 2 has been frozen at its value at the $D^{0} \bar{D}^{* 0}$ threshold because the $X$ (3872) signal happens around $\sigma_{1}$ within an energy region $|E| \ll \Delta$. As commented above we have checked that our results are stable if releasing it as in Eq. (C.6).

One can obtain an accurate numerical approximation to the exact expression for $M_{\mathrm{CDD}}^{(1)}$ in Eq. (C.4) (in the limit of $\Gamma_{R} \rightarrow 0^{+}$) if isospin breaking corrections in $\mu_{i}$ and $\beta_{i}$ are neglected (that are set equal to $\mu_{1}$ and $\beta_{1}$ ). These corrections are of $\mathcal{O}\left(\delta M / M_{D}\right)$, with $\delta M$ an isospin splitting mass in the $D^{(*)}$ multiplets. The resulting simplified expression is

$$
\begin{aligned}
M_{\mathrm{CDD}}^{(1)} & =\frac{E_{R} \pm 2 \alpha\left(\sqrt{\left|E_{R}\right|\left(\Delta-E_{R}\right)}+3 E_{R} / 2\right)}{1 \pm \alpha}, \\
\alpha & =\left(\frac{\Delta-E_{R}}{\left|E_{R}\right|}\right)^{\frac{1}{4}} .
\end{aligned}
$$

The + applies to the first solution and the - to the second. Substituting Eq. (C.9) in Eq. (C.5) we have the following expressions for $\widetilde{\lambda}$ and $\beta_{1}$ :

$$
\begin{aligned}
\tilde{\lambda} & =\frac{4 \mu \alpha^{2}}{\varkappa_{R}(1 \pm \alpha)^{2}}\left(E_{R}+\sqrt{\left|E_{R}\right|\left(\Delta-E_{R}\right)}\right)^{2}, \\
\beta_{1} & =\frac{2 \mu}{\varkappa_{R}(1 \pm \alpha)}\left(-E_{R} \pm \alpha \sqrt{\left|E_{R}\right|\left(\Delta-E_{R}\right)}\right),
\end{aligned}
$$

where $\varkappa_{R}=\varkappa_{1}$ and $\varkappa_{2}=\sqrt{2 \mu\left(\Delta-E_{R}\right)}$. From Eqs. (C.9) and (C.11) we also have an explicit expression for $M_{\mathrm{CDD}}$,

$$
\begin{aligned}
& M_{\mathrm{CDD}}=E_{R} \\
& +\frac{8 \mu \alpha^{2}\left(E_{R}+\sqrt{\left|E_{R}\right|\left(\Delta-E_{R}\right)}\right)^{2}}{4 \mu(1 \pm \alpha)\left(-E_{R} \pm \alpha \sqrt{\left|E_{R}\right|\left(\Delta-E_{R}\right)}\right)+\varkappa_{R}(1 \pm \alpha)^{2}\left(\varkappa_{R}+\varkappa_{2}\right)} .
\end{aligned}
$$

It is interesting to consider the limit $\alpha \rightarrow \infty$ because it is relevant for $X(3872)$ given the fact that $\left|E_{R}\right| \ll \Delta$ and Eqs. (C.9), (C.11) and (C.12) largely simplify. In this limit there is only one solution which is given by

$\beta=3 \varkappa_{2}$,

$\lambda=4 \varkappa_{R} \Delta$,

$M_{\mathrm{CDD}}=\frac{4}{3} \sqrt{\Delta\left|E_{R}\right|}$.

We can also see that in this limit there is a virtual state in the second RS, with similar energy as the bound state imposed by construction. Since $\varkappa_{R}$ is a root, and writing the three-momentum of the new solution as $i \varkappa_{2}$, we have from the secular equation the still exact relation

$\varkappa_{2}=-\varkappa_{R}-\frac{\left(\varkappa_{2}^{2}+2 \mu M_{\mathrm{CDD}}\right)}{\beta+\varkappa_{R}}$.

Now, implementing in this equation the values for the constants obtained in Eq. (C.13) we simply have, for $\alpha \rightarrow \infty$,

$\varkappa_{2}=-\frac{13}{9} \varkappa_{R}$.

Equation (C.14) considered for values of $\varkappa_{2}^{2}$ much larger than $2 \mu M_{\mathrm{CDD}}$ also implies that the third solution in this limit is $\varkappa_{3}=-\beta$.

To end this appendix let us discuss for $\alpha \rightarrow \infty$ how the poles move when including the finite width of $D^{* 0}$, that is, with $\Gamma_{*} \neq 0$. First, because of the condition imposed to guarantee the presence of the bound state with $\Gamma_{*}=0$, one 
can rewrite $\lambda$ as $\lambda=\left(\varkappa_{R}^{2} / 2 \mu+M_{\mathrm{CDD}}\right)\left(\beta+\varkappa_{R}\right)$. The secular equation to calculate its final pole position at $i \varkappa_{B}$ is then

$$
\begin{aligned}
& \left(\frac{\varkappa_{R}^{2}}{2 \mu}+M_{\mathrm{CDD}}\right)\left(\beta+\varkappa_{R}\right) \\
& -\left(\frac{\varkappa_{B}^{2}}{2 \mu}+M_{\mathrm{CDD}}+i \frac{\Gamma_{*}}{2}\right)\left(\beta+\varkappa_{B}\right)=0 .
\end{aligned}
$$

In the limit $\alpha \rightarrow \infty$ we can neglect $\varkappa_{R, B}$ in front of $\beta$ and the previous equation takes us to the solution

$\varkappa_{B}=\varkappa_{R}-i \mu \frac{\Gamma_{*}}{2 \varkappa_{R}}$.

The corresponding energy $E_{B}$ is

$E_{B}=-\frac{\varkappa_{B}^{2}}{2 \mu}-i \frac{\Gamma_{*}}{2}=-\frac{\varkappa_{R}^{2}}{2 \mu}=E_{R}$,

with quadratic terms in $\Gamma_{*}$ neglected both in Eqs. (C.17) and (C.18).

Let us move to calculate the pole position of the nearthreshold virtual state. Instead of Eq. (C.14) we now have the exact relation,

$\varkappa=-\varkappa_{R}+i \mu \frac{\Gamma_{*}}{-\varkappa+\varkappa_{R}}-\frac{\varkappa^{2}+i \mu \Gamma_{*}+2 \mu M_{\mathrm{CDD}}}{\beta+\varkappa_{R}}$.

The dominant contribution to the imaginary part stems from the second term on the right-hand side of the previous equation since $\beta \gg \varkappa_{R}$. We then have

$\varkappa_{2}=-\frac{13}{9} \varkappa_{R}-i \frac{9}{22} \frac{\mu \Gamma_{*}}{\varkappa_{R}}$.

The associated energy $E_{2}$ is

$E_{2}=-\frac{\varkappa_{2}^{2}}{2 \mu}-i \frac{\Gamma_{*}}{2}=\left(\frac{13}{9}\right)^{2} E_{R}+i \frac{\Gamma_{*}}{11}$,

where quadratic term in $\Gamma_{*}$ have been neglected. Notice how Eqs. (C.18) and (C.21) imply a much smaller imaginary part in absolute value for $E_{B}$ and $E_{2}$ than the half of the width of the constituent $D^{* 0}$, in agreement with the numerical results reported in Sect. 6.3.

For the deep virtual-state pole we consider again Eq. (C.19) and neglect $\varkappa_{R}$ and $\sqrt{2 \mu\left|M_{\mathrm{CDD}}\right|}$ in front of $\varkappa$ and $\beta$ (as $x_{3} \approx-\beta$ ). We then have the following equation for the solution $\varkappa_{3}$ that gives rise to the leading contribution to its imaginary part:

$\frac{\varkappa_{3}^{2}}{\beta}+\varkappa_{3}+i \mu \Gamma_{*}\left(\frac{1}{\varkappa_{3}}+\frac{1}{\beta}\right)=0$.
Neglecting quadratic terms in $\Gamma_{*}$ the imaginary part in $\varkappa_{3}$ cancels and we obtain again the same result as above with $\Gamma_{*}=0, \varkappa_{3}=-\beta$. Its energy $E_{3}$ is

$E_{3}=-\frac{\beta^{2}}{2 \mu}-i \frac{\Gamma_{*}}{2}$,

and its width is just determined by that of its constituent $D^{* 0}$. This result is as expected because this pole is a deep one that stems from the direct $D^{0} \bar{D}^{* 0}$ scattering, since at those energies the CDD pole contributions is negligible compared to $\beta$ as $\lambda / E \beta \simeq 2 \mu \lambda / \beta^{3} \propto \sqrt{\left|E_{R}\right| / \Delta}$ and tends to zero. This is not the case for the lighter poles because they are associated with the bare state with a small component of $D^{0} \bar{D}^{* 0}$.

Indeed one can easily calculate the residue of $t(E)$ in the variable $k$ at the bound-state pole position for $\Gamma_{*}=0$ and $\alpha \rightarrow \infty$ and apply Eq. (67). The following limit result is obtained:

$X=\frac{2}{11}$,

of similar size as those reported in Sect. 6.3. This finite small number is related to the fact that for $\left|E_{R}\right| \rightarrow 0$ also $M_{\mathrm{CDD}} \rightarrow$ 0 but the quotient $\left|E_{R}\right| / M_{\mathrm{CDD}}=3 / 4 \sqrt{E_{R} / \Delta} \rightarrow 0$, so that in relative terms the binding energy is much closer to zero than $M_{\mathrm{CDD}}$ for the limit $\alpha \rightarrow \infty$.

It is also worth remarking that for all the three poles the corrections in their pole positions as a function of $\Gamma_{*}$ are analytic because they are simple poles (isolated singularities).

\section{References}

1. C. Hanhart, Y.S. Kalashnikova, A.E. Kudryavtsev, A.V. Nefediev, Phys. Rev. D 76, 034007 (2007). arXiv:0704.0605 [hep-ph]

2. E. Braaten, M. Lu, Phys. Rev. D 76, 094028 (2007). arXiv:0709.2697 [hep-ph]

3. E. Braaten, J. Stapleton, Phys. Rev. D 81, 014019 (2010). arXiv:0907.3167 [hep-ph]

4. O. Zhang, C. Meng, H.Q. Zheng, Phys. Lett. B 680, 453 (2009). arXiv:0901.1553 [hep-ph]

5. Y.S. Kalashnikova, A.V. Nefediev, Phys. Rev. D 80, 074004 (2009). arXiv:0907.4901 [hep-ph]

6. G.Y.Chen, W.S. Huo, Q. Zhao, Chin. Phys. C39(9), 093101 (2015). arXiv:1309.2859 [hep-ph]

7. V. Baru, J. Haidenbauer, C. Hanhart, Y. Kalashnikova, A.E. Kudryavtsev, Phys. Lett. B 586, 53 (2004). arXiv:hep-ph/0308129

8. X.W. Kang, Z.H. Guo, J.A. Oller, Phys. Rev. D 94(1), 014012 (2016). arXiv:1603.05546 [hep-ph]

9. L. Castillejo, R.H. Dalitz, F.J. Dyson, Phys. Rev. 101, 453 (1956)

10. S. Weinberg, Phys. Rev. 137, B672 (1965)

11. V. Baru, C. Hanhart, Y.S. Kalashnikova, A.E. Kudryavtsev, A.V. Nefediev, Eur. Phys. J. A 44, 93 (2010). arXiv:1001.0369 [hep-ph]

12. P. Artoisenet, E. Braaten, D. Kang, Phys. Rev. D 82, 014013 (2010). arXiv:1005.2167 [hep-ph]

13. C. Hanhart, Y.S. Kalashnikova, A.V. Nefediev, Eur. Phys. J. A 47, 101 (2011). arXiv:1106.1185 [hep-ph] 
14. B. Aubert et al. (BaBar Collaboration), Phys. Rev. D 77, 111101 (2008). arXiv:0803.2838 [hep-ex]

15. B. Aubert et al. (BaBar Collaboration), Phys. Rev. D 77, 011102 (2008). arXiv:0708.1565 [hep-ex]

16. I. Adachi et al. (Belle Collaboration), arXiv:0809.1224 [hep-ex]

17. T. Aushev et al. (Belle Collaboration), Phys. Rev. D 81, 031103 (2010). arXiv:0810.0358 [hep-ex]

18. T. Aaltonen et al. (CDF Collaboration), Phys. Rev. Lett. 103, 152001 (2009). arXiv:0906.5218 [hep-ex]

19. C. Patrignani et al. (Particle Data Group), Chin. Phys. C 40, 100001 (2016)

20. E. Braaten, M. Kusunoki, Phys. Rev. D 72, 054022 (2005). arXiv:hep-ph/0507163

21. M.B. Voloshin, Phys. Lett. B 579, 316 (2004). arXiv:hep-ph/0309307

22. C. Hanhart, Y.S. Kalashnikova, A.V. Nefediev, Phys. Rev. D 81, 094028 (2010). arXiv:1002.4097 [hep-ph]

23. E.M. Aitala et al. (E791 Collaboration), Phys. Rev. Lett. 86, 770 (2001). arXiv:hep-ex/0007028

24. J.A. Oller, Final state interactions in hadronic D decays. Phys. Rev. D 71, 054030 (2005). doi:10.1103/PhysRevD.71.05403. arXiv:hep-ph/0411105

25. J.A. Oller, in Proceedings of the Second Workshop on the CKM Unitarity Triangle, eConf C 0304052, WG412 (2003). arXiv:hep-ph/0306294

26. D.V. Bugg, Phys. Lett. B 572, 1 (2003) [(E) Phys. Lett. B 595, 556 (2004)]

27. J.A. Oller, Phys. Lett. B 477, 187 (2000). arXiv:hep-ph/9908493

28. S. Gardner, U.G. Meißner, Phys. Rev. D 65, 094004 (2002). arXiv:hep-ph/0112281

29. R. Aaij et al. (LHCb Collaboration), Eur. Phys. J. C 72, 1972 (2012). arXiv:1112.5310 [hep-ex]

30. J.A. Oller, E. Oset, Phys. Rev. D 60, 074023 (1999). arXiv:hep-ph/9809337

31. Z.H. Guo, J.A. Oller, Phys. Rev. D 93(5), 054014 (2016). arXiv:1601.00862 [hep-ph]

32. M.P. Valderrama, Phys. Rev. D 85, 114037 (2012). doi:10.1103/ PhysRevD.85.114037. arXiv:1204.2400 [hep-ph]

33. M. Suzuki, Phys. Rev. D 72, 114013 (2005). arXiv:hep-ph/0508258

34. L. Alvarez-Ruso, J.A. Oller, J.M. Alarcon, Phys. Rev. D 80, 054011 (2009). arXiv:0906.0222 [hep-ph]

35. L. Alvarez-Ruso, J.A. Oller, J.M. Alarcon, Phys. Rev. D 82, 094028 (2010). arXiv:1007.4512 [hep-ph]

36. C. Hanhart, Y.S. Kalashnikova, P. Matuschek, R.V. Mizuk, A.V. Nefediev, Q. Wang, Phys. Rev. Lett. 115(20), 202001 (2015). arXiv:1507.00382 [hep-ph]

37. F.-K. Guo, C. Hanhart, Y.S. Kalashnikova, P. Matuschek, R.V. Mizuk, A.V. Nefediev, Q. Wang, J.-L. Wynen, Phys. Rev. D 93(7), 074031 (2016). arXiv:1602.00940 [hep-ph]

38. E. Braaten, M. Lu, Phys. Rev. D 77, 014029 (2008). arXiv:0710.5482 [hep-ph]

39. F. James, MINUIT-Function Minimization and Error Analysis. CERN Program Library Long Writeup D506, Version 94.1

40. T. Hyodo, D. Jido, A. Hosaka, Phys. Rev. C 85, 015201 (2012). arXiv:1108.5524 [nucl-th]

41. F. Aceti, E. Oset, Phys. Rev. D 86, 014012 (2012). arXiv: 1202.4607 [hep-ph]

42. T. Sekihara, T. Hyodo, D. Jido, PTEP 2015, 063 D04 (2015). arXiv:1411.2308 [hep-ph]
43. C. Hanhart, J.R. Pelaez, G. Rios, Phys. Rev. Lett. 100, 152001 (2008). arXiv:0801.2871 [hep-ph]

44. M. Albaladejo, J.A. Oller, Phys. Rev. D 86, 034003 (2012). arXiv:1205.6606 [hep-ph]

45. C. Hanhart, J.R. Pelaez, G. Rios, Phys. Lett. B 739, 375 (2014). arXiv: 1407.7452 [hep-ph]

46. T. Hyodo, Phys. Rev. C 90(5), 055208 (2014). arXiv:1407.2372 [hep-ph]

47. V. Bernard, D. Hoja, U.-G. Meissner, A. Rusetsky, JHEP 0906, 061 (2009). arXiv:0902.2346 [hep-lat]

48. T. Ledwig, V. Pascalutsa, M. Vanderhaeghen, Phys. Rev. D 82, 091301 (2010). arXiv:1004.5055 [hep-ph]

49. F.-K. Guo, C. Hanhart, F.J. Llanes-Estrada, U.-G. Meissner, Phys. Lett. B 703, 510 (2011). doi:10.1016/j.physletb.2011.08.022. arXiv:1105.3366 [hep-lat]

50. F.K. Guo, U.G. Meissner, Phys. Rev. Lett. 109, 062001 (2012). arXiv:1203.1116 [hep-ph]

51. E. Cincioglu, J. Nieves, A. Ozpineci, A.U. Yilmazer, Eur. Phys. J. C 76(10), 576 (2016). arXiv:1606.03239 [hep-ph]

52. P.G. Ortega, J. Segovia, D.R. Entem, F. Fernandez, Phys. Rev. D 81, 054023 (2010). arXiv:1001.3948 [hep-ph]

53. D.R. Entem, P.G. Ortega, F. Fernandez, AIP Conf. Proc. 1735, 060006 (2016). arXiv:1601.03901 [hep-ph]

54. C. Meng, J.J. Sanz-Cillero, M. Shi, D.L. Yao, H.Q. Zheng, Phys. Rev. D 92(3), 034020 (2015). arXiv:1411.3106 [hep-ph]

55. C. Meng, K.T. Chao, Phys. Rev. D 75, 114002 (2007) arXiv:hep-ph/0703205

56. J. Ferretti, G. Galat, E. Santopinto, Phys. Rev. C 88(1), 015207 (2013). arXiv:1302.6857 [hep-ph]

57. D. Morgan, Nucl. Phys. A 543, 632 (1992)

58. S.K. Choi et al. (Belle Collaboration), Phys. Rev. Lett. 91, 262001 (2003). arXiv:hep-ex/0309032

59. R.F. Lebed, R.E. Mitchell, E.S. Swanson, Prog. Part. Nucl. Phys. 93, 143 (2017). arXiv:1610.04528 [hep-ph]

60. D. Gamermann, E. Oset, Eur. Phys. J. A 33, 119 (2007). arXiv:0704.2314 [hep-ph]

61. D. Gamermann, J. Nieves, E. Oset, E.R. Arriola, Phys. Rev. D 81, 014029 (2010). arXiv:0911.4407 [hep-ph]

62. S. Fleming, M. Kusunoki, T. Mehen, U. van Kolck, Phys. Rev. D 76, 034006 (2007). arXiv:hep-ph/0703168

63. Y. Liu, I. Zahed, Int. J. Mod. Phys. E 26(01, 02), 1740017 (2017). arXiv:1610.06543 [hep-ph]

64. A. Sibirtsev, J. Haidenbauer, S. Krewald, U.G. Meißner, A.W. Thomas, Phys. Rev. D 71, 054010 (2005). arXiv:hep-ph/0411386

65. X.W. Kang, J. Haidenbauer, U.G. Meißner, JHEP 1402, 113 (2014). arXiv:1311.1658 [hep-ph]

66. X.W. Kang, J. Haidenbauer, U.G. Meißner, Phys. Rev. D 91(7), 074003 (2015). arXiv:1502.00880 [nucl-th]

67. Y.F. Liu, X.W. Kang, Symmetry 8(3), 14 (2016)

68. M. Padmanath, C.B. Lang, S. Prelovsek, Phys. Rev. D 92(3), 034501 (2015). arXiv:1503.03257 [hep-lat]

69. S.H. Lee et al. (Fermilab Lattice and MILC Collaborations), arXiv:1411.1389 [hep-lat]

70. S. Prelovsek, L. Leskovec, Phys. Rev. Lett. 111, 192001 (2013). arXiv:1307.5172 [hep-lat] 